\title{
Rigorous derivation of the thin film approximation with roughness-induced correctors*
}

\author{
Laurent Chupin ${ }^{\dagger} \quad$ Sébastien Martin ${ }^{\ddagger}$
}

April 2012

\begin{abstract}
We derive the thin film approximation including roughness-induced correctors. This corresponds to the description of a confined Stokes flow whose thickness is of order $\varepsilon \ll 1$; but we also take into account the roughness patterns of the boundary that are described at order $\varepsilon^{2}$, leading to a perturbation of the classical Reynolds approximation. The asymptotic expansion leading to the description of the scale effects is rigorously derived, through a sequence of Reynolds-type problems and Stokes-type boundary layer problems. Well-posedness of the related problems and estimates in suitable functional spaces are proven, at any order of the expansion. In particular, we show that the micro-/macro-scale coupling effects may be analyzed as the consequence of two features: the interaction between the macroscopic scale (order 1 ) of the flow and the microscopic scale (order $\varepsilon$ ) is perturbed by the interaction with a microscopic scale of order $\varepsilon^{2}$ related to the roughness patterns. Moreover, the converging-diverging profile of the confined flow, which is typical in lubrication theory provides additional micro-macro-scales coupling effects.
\end{abstract}

${ }^{*}$ This work was supported by the ANR project ANR-08-JCJC-0104-01 : RUGO (Analyse et calcul des effets de rugosités sur les écoulements). The authors are very grateful to David Gérard-Varet for fruitful discussions on the subject of this paper.

${ }^{\dagger}$ Université Blaise Pascal, Clermont-Ferrand II, Laboratoire de Mathématiques CNRSUMR 6620, Campus des Cézeaux, F-63177 Aubière cedex, France.

laurent. chupin@math. univ-bpclermont.fr

‡Université Paris-Sud XI, Laboratoire de Mathématiques CNRS-UMR 8628, Faculté des Sciences d'Orsay, Bât. 425, F-91405 Orsay cedex, France.

sebastien.martin@math.u-psud.fr 


\section{Contents}

1 Introduction 3

1.1 General framework . . . . . . . . . . . . . . . . . . . . 3

1.2 Position of the problem . . . . . . . . . . . . 4

2 Asymptotic expansion: ansatz and intermediate problems $\quad 6$

2.1 Notations . . . . . . . . . . . . . . . . . 6

2.2 Ansatz . . . . . . . . . . . . . . . . . . . . . . . . . . . 9

2.3 Order 0 and first corrections . . . . . . . . . . . 10

2.4 Higher orders of the asymptotic expansion . . . . . . . . . . 13

3 Mathematical results related to the different scale problems $\mathbf{1 5}$

3.1 Stokes problems: well-posedness and behavior of the solutions . . 15

3.1.1 Initialization step: analysis of problem $\left(\mathrm{S}^{(1)}\right) \ldots \ldots \ldots$

3.1.2 Induction step: analysis of problem $\left(\mathrm{S}^{(j)}\right)$ for $j \geq 2 \ldots 17$

3.2 Well-posedness of the Reynolds problem . . . . . . . . . 18

3.3 Algorithm . . . . . . . . . . . . . . . . . 20

4 Error analysis $\quad 21$

4.1 Lift procedure . . . . . . . . . . . . . . . . . . 23

4.1.1 Lift velocity at the boundary . . . . . . . . . . . . 23

4.1.2 Lift velocity using the Bogovskii formulae . . . . . . . . . 23

4.2 Classical Stokes estimates . . . . . . . . . . . . . . . 24

4.3 Explicit estimates with respect to $\varepsilon \ldots \ldots \ldots \ldots \ldots$

4.3.1 Control of the source terms . . . . . . . . . . 26

4.3 .2 Boundary lift . . . . . . . . . . . . . . . 26

4.3 .3 Bogovskii lift . . . . . . . . . . . . . . . 27

4.3 .4 Estimates . . . . . . . . . . . . . . . . 27

4.4 Error analysis on adapted spaces $\ldots \ldots \ldots \ldots . \ldots 27$

5 Discussion on the different scale effects 28

5.1 Contribution of the rugosities in the thin film approximation . . 29

5.2 Multiscale coupling effects due to the curvature of the film thickness 30

$\begin{array}{ll}\text { A Proof of Lemma } 3.3 & 31\end{array}$

B Proof of Lemma 3.5 33

C Proof of Lemma 3.7 35

D Proof of Proposition 4.4 36

$\begin{array}{ll}\text { E Proof of Proposition 4.5 } & 37\end{array}$

$\begin{array}{lll}\text { F } & \text { Proof of Lemma } 4.8 & 37\end{array}$ 


\section{Introduction}

\subsection{General framework}

Lubricated flows are very present in today's world: from the journal bearings to the computer disk drives through the microfluid or in biofluid mechanics. The first relevant model for such thin flows was proposed by O. Reynolds in 1886, see [24]. From a mathematical point of view, the rigorous justification of the Reynolds equation from the Stokes equation is due to G. Bayada and M. Chambat [4]. Other studies have further refined this result, especially those of S. Nazarov [22] and more recently J. Wilkening [27] who provides practical error estimates at any order with an extensive description of the constants with respect to the normalized gap.

From another point of view, many studies investigate the effect of wall roughness on Newtonian flows. In 1827, Navier [21] was one of the first scientists to note that the roughness could drag a fluid. Since then, numerous studies attempted to prove mathematical results in this direction, see for instance the works of W. Jäger and A. Mikelic [18], Y. Amirat and co-authors [1, 2] and more recently the works of D. Bresch and V. Milisic [10]. Note that all these works formulate the roughness using a periodic function (whose amplitude and period are supposed to be small). In a context of more general "roughness" patterns, there exists similar recent results, see [3, 15].

Numerous works focus on the combination of the two phenomena: lubrication and roughness. Let us mention the seminal works by H.G. Elrod [13] who used a formal double-variable technique applied to the so-called Reynolds roughness. With a different scaling of the roughness, D.-C. Sun and K.-K Chen [26] formally derive the effect of the so-called Stokes roughness in the framework of lubrication. In [23], N. Phan-Thien generalized these approaches by describing the perturbation solution to the Reynolds and Stokes equations for a two-dimensional slider bearing with homogeneous surface roughness. However, the scalings that were previously considered are different from the one that we address.

From a mathematical point of view, analytical studies have been led for various situations: in the papers by I. Ciuperca and co-authors [5, 7], the size of the roughness is assumed to be at least of the same order as the thickness of the film. In [9], D. Bresch and co-authors consider the case where the roughness is assumed to be small compared to the thickness of the flow (which is the case in the present paper) but they show a convergence in a rescaled domain, that focuses on the roughness effect. Recently, in [11], J. Casado-Díaz and co-authors proposed a relatively general study (in terms of orders of magnitude of roughness and thickness of the domain). However, their article is entirely focused on the wall laws which is not our point of view in this paper.

In this paper, we focus on flows in a thin domain (with thickness $\varepsilon \ll 1$ ) which is rough. The size of the roughness is assumed to be of size $\varepsilon^{2}$ which is physically realistic, see for example [19]. By separating the effects due to lubrication and those due to the roughness, we present and rigorously justify 
an asymptotic expansion with respect to $\varepsilon$. The development is done at any order, so that we are guaranteed to be optimal with respect to the truncation error. We also highlight the particular effects of roughness, and the multiscale coupling effects of the curvature of the macroscopic domain (which cannot be neglected in lubricated devices).

Several relevant questions are not addressed in this article. First, concerning the choice of orders of magnitude for the thickness of the fluid and the roughness ( $\varepsilon$ and $\varepsilon^{2}$ in this article). It seems fairly sensible to believe that the proposed method can be adapted to cases where the thickness of the fluid is of order $\varepsilon$, while the roughness is of the order $\varepsilon^{\alpha}$, with $\alpha>1$. Nevertheless, the ansatz will be different, depending on $\alpha$. Second, recent works on random roughness, see $[3,15]$, could make us think that our results can be extended to more general cases of roughness. In fact, the construction of our development strongly depends on the behavior of solutions of the Stokes equation on a half-space, whose lower boundary is periodic. The behavior of such solutions must be sufficiently decreasing at infinity to justify our development. Unfortunately, it seems that this decrease is only logarithmic in the case of a random boundary (while it is exponential in our periodic case). Besides, another task related to the regularity of the roughness patterns is not addressed in this paper: what is the behavior of the solution when the patterns are not Lipschitz continuous? In particular, what is the influence of roughness jump discontinuities over the flow?

\subsection{Position of the problem}

We consider the flow of a viscous incompressible fluid in a domain of $\mathbb{R}^{d+1}$, $d \in \mathbb{N}\{0\}$. Mathematically, we take into account the thickness and the roughness by introducing a small parameter $\varepsilon>0$ and defining a time dependent domain

$$
\Omega_{\varepsilon}(t)=\left\{(\mathbf{x}, y) \in \mathbb{T}^{d} \times \mathbb{R}, \quad-\varepsilon^{2} h^{-}\left(\frac{\mathbf{x}-\mathbf{s} t}{\varepsilon^{2}}\right)<y<\varepsilon h^{+}(\mathbf{x})\right\} .
$$

Here, $\mathbb{T}^{d}:=(\mathbb{R} / \mathbb{Z})^{d}$ denotes the torus; the two functions $h^{+}$and $h^{-}$are supposed to be smooth, positive and 1-periodic in each variable of $\mathbb{R}^{d}$ (we will say $\mathbf{x}$-periodic). In this prospect $\varepsilon^{-2}$ is considered to be an integer. The vector $\mathbf{s} \in \mathbb{R}^{d}$ denotes the shear velocity of the device (velocity of the lower rigid surface). Moreover, the upper (resp. lower) boundary of the domain $\Omega_{\varepsilon}(t)$ is denoted $\Gamma_{\varepsilon}^{+}$(resp. $\left.\Gamma_{\varepsilon}^{-}\right)$. A typical situation describing the scaling orders is illustrated on figure 1. For the sake of simplicity, we have assumed that there is no oscillation at the upper boundary, even if our methodology applies when considering roughness patterns on the upper boundary (with the same wavelength and amplitude).

Stokes equations express, in particular, the momentum conservation connecting the velocity field $\mathbf{U}=(\mathbf{u}, v)$ to the pressure $p$. These equations must 


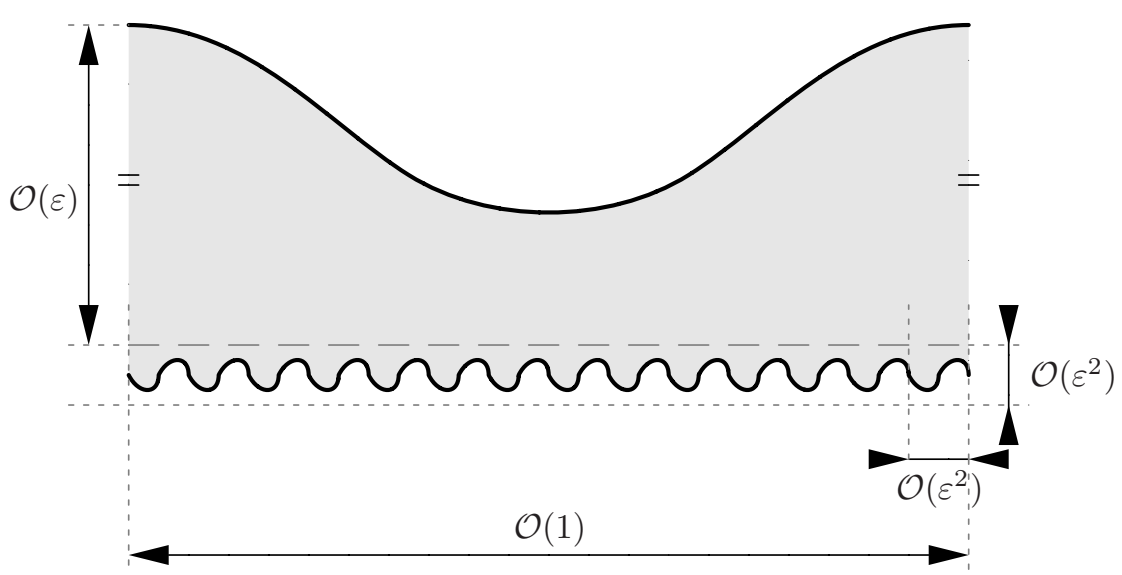

Figure 1: Geometry of the Stokes flow: thin film assumption and roughness patterns.

be supplemented by no-slip boundary conditions:

$$
\left\{\begin{aligned}
-\Delta \mathbf{U}+\nabla p & =0, & & \text { in } \Omega_{\varepsilon}(t), \\
\operatorname{div} \mathbf{U} & =0, & & \text { in } \Omega_{\varepsilon}(t), \\
\mathbf{U} & =\mathbf{0}, & & \text { on } \Gamma_{\varepsilon}^{+}(t), \\
\mathbf{U} & =(\mathbf{s}, 0), & & \text { on } \Gamma_{\varepsilon}^{-}(t) .
\end{aligned}\right.
$$

In the sequel, for the sake of simplicity, we may omit the variable $t$ although the domain depends on time as a parameter.

It is well-known (see [4] or more recently [27]) that the solutions of the Stokes system in a thin confined domain with a flat bottom are approached by those of the Reynolds equation. More precisely, under the thin film assumption, assuming that the bottom is flat, i. e. $h^{-}=0$, the flow is governed by:

$$
\begin{gathered}
\mathbf{u}^{+}(\mathbf{x}, y)=\mathbf{u}_{0}\left(\mathbf{x}, \frac{y}{\varepsilon}\right)+\mathcal{O}\left(\varepsilon^{2}\right), \quad v^{+}(\mathbf{x}, y)=\varepsilon v_{0}\left(\mathbf{x}, \frac{y}{\varepsilon}\right)+\mathcal{O}\left(\varepsilon^{3}\right), \\
p^{+}(\mathbf{x}, y)=\frac{p_{0}(\mathbf{x})}{\varepsilon^{2}}+\mathcal{O}(1)
\end{gathered}
$$

where $\mathbf{u}_{0}, v_{0}$ and $p_{0}$ correspond to the rescaled velocity field and pressure at main order. It can be shown that $p_{0}$, which only depends on the variable $\mathbf{x}$, is the unique solution (defined up to an additive constant) of the Reynolds equation; besides, the velocity can be deduced from the pressure by means of a straightforward integration.

In this context, we aim at describing the corresponding correction due to the the rough boundary at main order. As an illustration, numerical experiments, see figures 2 to 5 , have been performed with FreeFem ${ }^{++}{ }^{1}$, based on the finite element

$1_{\text {www: //freefem.org }}$ 
method. In particular, we have used a $\left(\mathbb{P}_{2}, \mathbb{P}_{2}, \mathbb{P}_{1}\right)$ finite element decomposition, which satisfies the discrete inf-sup condition, leading to a well-posed discretized problem. Numerical results highlight the differences in terms of computational costs and they give a preview of the perturbations due to the roughness patterns compared to a smooth boundary, which gives insight into the boundary layer structure and its interaction with the main flow.

The paper is organized as follows:

In section 2, we present the formal asymptotic expansion, based on a sequence of functions which alternatively satisfy a Reynolds-type problem and a corrective Stokes problem (defined in a semi-infinite boundary layer domain). In section 3, we present the well-posedness of the intermediate problems and analyze the behavior of the solutions. Moreover, we establish an algorithm related to the computation of the approximation of the solution, at any order. In particular, we show that each problem only depends on the previous ones, although this property is not clear at first glance! section 4 is devoted to the error analysis which, in the end, rigorously justifies the asymptotic expansion, see Theorem 4.6. In section 5, we focus on the coupling scale effects: we present quantitative comparison in the case with or without roughness correction, emphasizing the fact that the converging-diverging profile of the lubricated space is a a real source of complexity. Finally, some technical proofs are postponed in the Appendixes.

\section{Asymptotic expansion: ansatz and intermedi- ate problems}

\subsection{Notations}

With the sight of the different scales in the domain, we split the domain $\Omega_{\varepsilon}(t)$ into three parts : $\Omega_{\varepsilon}(t)=\Omega_{\varepsilon}^{-}(t) \cup \Gamma \cup \Omega_{\varepsilon}^{+}$where $\Omega_{\varepsilon}^{-}(t)$ and $\Omega_{\varepsilon}^{+}$are defined by

$$
\begin{gathered}
\Omega_{\varepsilon}^{+}=\left\{(\mathbf{x}, y) \in \mathbb{T}^{d} \times \mathbb{R}, \quad 0<y<\varepsilon h^{+}(\mathbf{x})\right\}, \\
\Omega_{\varepsilon}^{-}(t)=\left\{(\mathbf{x}, y) \in \mathbb{T}^{d} \times \mathbb{R}, \quad-\varepsilon^{2} h^{-}\left(\frac{\mathbf{x}-\mathbf{s} t}{\varepsilon^{2}}\right)<y<0\right\},
\end{gathered}
$$

and the boundary $\Gamma$ connecting the two subdomains is defined by $\Gamma=\mathbb{T}^{d} \times\{0\}$.

The first step of the construction of the ansatz is to notice that the flow is controlled by that in the domain $\Omega_{\varepsilon}^{+}$which is of order $\varepsilon$ with respect to the vertical coordinate, and that the flow in the domain $\Omega_{\varepsilon}^{-}(t)$, which is of order $\varepsilon^{2}$ in both horizontal and vertical directions, can induce a correction. Let us define the two rescaled subdomains. As a matter of fact, the main flow is governed by the Reynolds thin film flow, based on the changes of variables

$$
Z:=\frac{y}{\varepsilon} .
$$



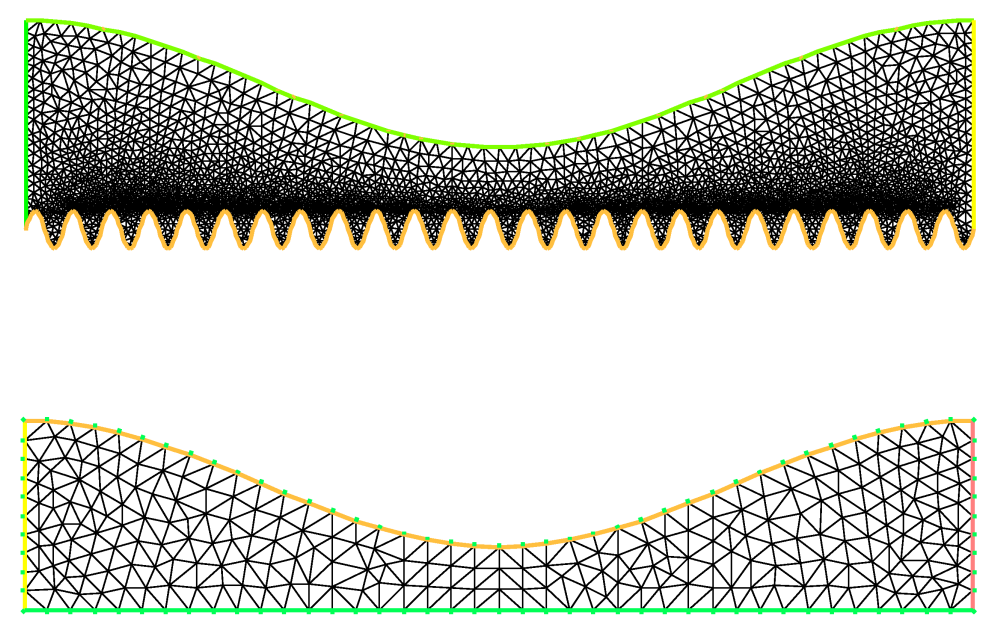

Figure 2: Mesh used for the computations of the solution of the Stokes system (approximation with the $\left(P_{2}, P_{2}, P_{1}\right)$ finite element approximation). Domain with roughness patterns or with smooth boundary.
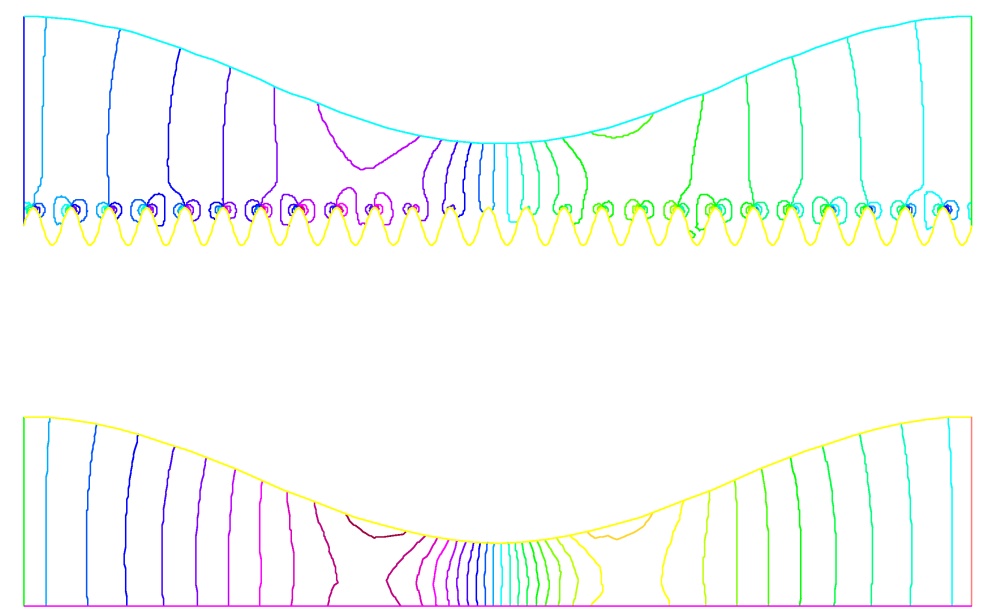

Figure 3: Pressure distribution in the domain with/without roughness patterns. 

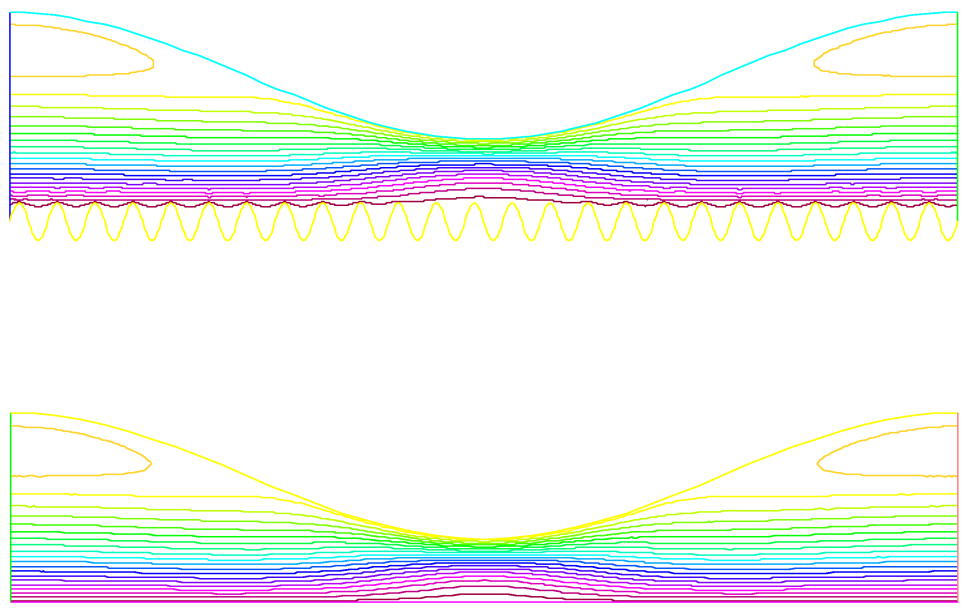

Figure 4: Horizontal velocity distribution in the domain with/without roughness patterns.
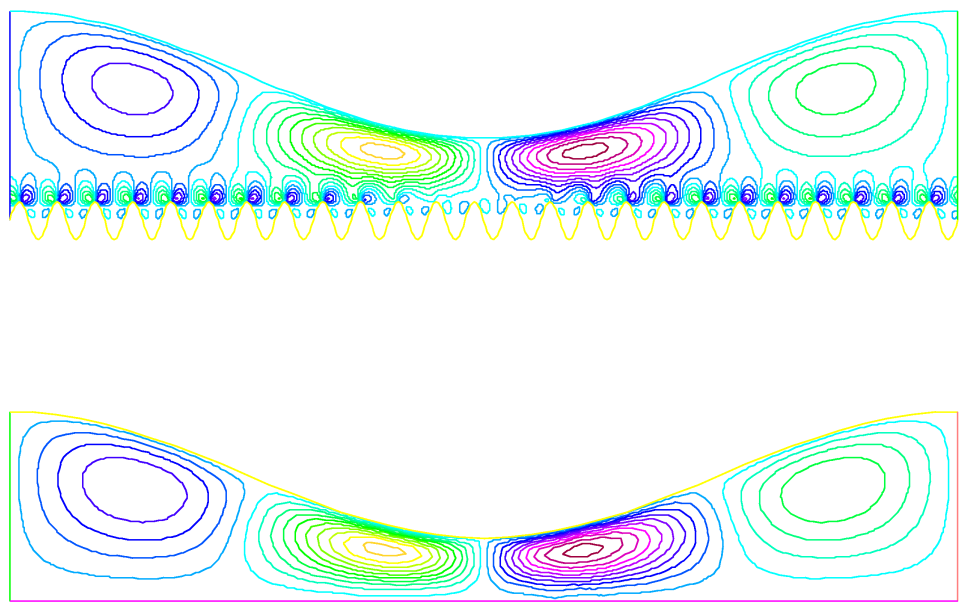

Figure 5: Vertical velocity distribution in the domain with/without roughness patterns. 
Due to the consideration of the roughness patterns, the boundary layer is rescaled by the homothetic transformation

$$
\mathbf{X}:=\frac{\mathbf{x}}{\varepsilon^{2}}, \quad Y:=\frac{y}{\varepsilon^{2}}, \quad T:=\frac{t}{\varepsilon^{2}} .
$$

Definition 2.1 We define the following rescaled domains:

- The Reynolds domain is defined by

$$
\omega_{R}:=\left\{(\mathbf{x}, Z) \in \mathbb{T}^{d} \times \mathbb{R}, \quad 0<Z<h^{+}(\mathbf{x})\right\},
$$

whose the upper and lower boundaries are respectively denoted $\gamma_{+}$and $\gamma_{0}$.

- The boundary layer domain is defined by

$$
\omega_{b l}(T)=\left\{(\mathbf{X}, Y) \in \mathbb{T}^{d} \times \mathbb{R}, \quad-h^{-}(\mathbf{X}-\mathbf{s} T)<Y\right\},
$$

whose the lower boundary is denoted $\gamma_{b l}(T)$.

Notice that the boundary layer does depend on time, as this subdomain has a moving boundary which emerges from the roughness patterns of the lower surface and the shear velocity of this surface. Actually, time-dependant boundary conditions leads us to define a more suitable rescaled variable which takes into account the shear effects and the adhering conditions that relate time and space variables. Notice that time-dependency of the boundary layer is taken into account in the space variable as a simple parameter, which allows us to insist on the instantaneity of the Stokes system, even at this rescaled level.

\subsection{Ansatz}

We propose the following asymptotic expansion

$$
(\mathbf{u}, v, p):=\left(\mathbf{u}^{(N)}, v^{(N)}, p^{(N)}\right)+\left(\boldsymbol{\mathcal { R }}^{(N)}, \mathcal{S}^{(N)}, \mathcal{Q}^{(N)}\right)
$$

with the following partial sums:

$$
\begin{aligned}
& \mathbf{u}^{(N)}(\mathbf{x}, y, t)=\sum_{j=0}^{N} \varepsilon^{j}\left[\mathbf{u}_{j}\left(\mathbf{x}, \frac{y}{\varepsilon}\right)+\varepsilon \widetilde{\mathbf{u}}_{j+1}\left(\mathbf{x}, \frac{\mathbf{x}-\mathbf{s} t}{\varepsilon^{2}}, \frac{y}{\varepsilon^{2}}\right)\right], \\
& v^{(N)}(\mathbf{x}, y, t)=\sum_{j=0}^{N} \varepsilon^{j+1}\left[v_{j}\left(x, \frac{y}{\varepsilon}\right)+\widetilde{v}_{j+1}\left(\mathbf{x}, \frac{\mathbf{x}-\mathbf{s} t}{\varepsilon^{2}}, \frac{y}{\varepsilon^{2}}\right)\right], \\
& p^{(N)}(\mathbf{x}, y, t)=\sum_{j=0}^{N} \varepsilon^{j-2}\left[p_{j}\left(\mathbf{x}, \frac{y}{\varepsilon}\right)+\varepsilon \widetilde{p}_{j+1}\left(\mathbf{x}, \frac{\mathbf{x}-\mathbf{s} t}{\varepsilon^{2}}, \frac{y}{\varepsilon^{2}}\right)\right] .
\end{aligned}
$$

Each term of this expansion corresponds to the solution of a Reynolds problem or Stokes problem. More precisely, we will see that $\left(\mathbf{u}_{j}, v_{j}, p_{j}\right)$ is the solution of a Reynolds-type problem. This solution being extended in the boundary layer, this leads to a perturbation of the no-slip boundary condition on the shearing 
(bottom) surface. Thus, the exact boundary condition is not satisfied and we have to impose a correction; this is the role of $\left(\widetilde{\mathbf{u}}_{j+1}, \widetilde{v}_{j+1}, \widetilde{p}_{j+1}\right)$ which is the solution of a Stokes problem in a semi-infinite domain. As a consequence, the behavior of the Stokes solution is such that it defines a perturbation of the zero no-slip boundary condition at the top of the domain and, thus, this will be taken into account in the definition of the elementary solution at next order, in order to balance all the effects related to the successive perturbations of the flow and boundary conditions.

Let us mention that the expansion includes a remainder $\left(\mathcal{R}^{(N)}, \mathcal{S}^{(N)}, \mathcal{Q}^{(N)}\right)$ which, by means of subtraction, is proven to satisfy a Stokes problem in the "physical" domain. A major task of this work is to derive some bounds on this remainder w.r.t. $\varepsilon$ in order to prove in a rigorous way that the asymptotic expansion is valid.

Before describing the systems satisfied by the previous terms, let us highlight that difficulties are twofold: not only well-posedness of the elementary problems is a major task, but also suitable definition of these elementary problems is crucial: in the range of difficulty, it can be viewed as the most important point of the analysis, as it enhances to include all the corrective properties of the expansion by keeping the well-posedness properties of the elementary problems and feasibility of a numerical procedure (algorithm) for the computation of the solution.

\subsection{Order 0 and first corrections}

We put the ansatz (2), into the Stokes system (1), and then identify the terms of same order with respect to $\varepsilon$.

○ Main flow at scale $\varepsilon^{0}$ : Reynolds flow in the thin film domain. The functions $\left(\mathbf{u}_{0}, v_{0}, p_{0}\right)$ satisfy the classical Reynolds problem

$$
\left(\mathrm{R}^{(0)}\right)\left\{\begin{aligned}
-\partial_{Z}^{2} \mathbf{u}_{0}+\nabla_{\mathbf{x}} p_{0} & =-\boldsymbol{A}_{0}, & & \text { on } \omega_{R}, \\
\partial_{Z} p_{0} & =-B_{0}, & & \text { on } \omega_{R}, \\
\operatorname{div}_{\mathbf{x}} \mathbf{u}_{0}+\partial_{Z} v_{0} & =-C_{0}, & & \text { on } \omega_{R}, \\
\left(\mathbf{u}_{0}, v_{0}\right) & =(\mathbf{s}, 0), & & \text { on } \gamma_{0} \\
\left(\mathbf{u}_{0}, v_{0}\right) & =\left(\mathbf{0},-\beta_{1}\right) & & \text { on } \gamma_{+},
\end{aligned}\right.
$$

where the functions $\boldsymbol{A}_{0}, B_{0}$ and $C_{0}$ only depend on the variable $\mathbf{x}$. We will see, a posteriori, that $\boldsymbol{A}_{0}=\mathbf{0}, B_{0}=0$ and $C_{0}=0$. Coefficient $\beta_{1}$ will be related to the corrective procedure (although it will be proven to be independent from the corrective procedure), see Remark 2.5.

$\circ$ First correction at scale $\varepsilon^{1}$ : Stokes flow in the boundary layer. We first set $T=0$ (the boundary problem can be defined in a similar way for any time $T \neq 0)$. The functions $\left(\widetilde{\mathbf{u}}_{1}, \widetilde{v}_{1}, \widetilde{p}_{1}\right)$ satisfy a Stokes problem: 


$$
\left(\mathrm{S}^{(1)}\right)\left\{\begin{aligned}
-\Delta_{\mathbf{X}} \widetilde{\mathbf{u}}_{1}-\partial_{Y}^{2} \widetilde{\mathbf{u}}_{1}+\nabla_{\mathbf{X}} \widetilde{p}_{1} & =\mathbf{0}, & & \text { on } \omega_{b l}(0), \\
-\Delta_{\mathbf{X}} \widetilde{v}_{1}-\partial_{Y}^{2} \widetilde{v}_{1}+\partial_{Y} \widetilde{p}_{1} & =B_{0}, & & \text { on } \omega_{b l}(0), \\
\operatorname{div} \widetilde{\mathbf{u}}_{1}+\partial_{Y} \widetilde{v}_{1} & =0, & & \text { on } \omega_{b l}(0), \\
\left(\widetilde{\mathbf{u}}_{1}, \widetilde{v}_{1}\right) & =\left(\widetilde{\mathcal{U}}_{1}, \widetilde{\mathcal{V}}_{1}\right), & & \text { on } \gamma_{b l}(0),
\end{aligned}\right.
$$

where the source term in the boundary condition should be read as

$$
\tilde{\mathcal{U}}_{1}: \mathbf{X} \rightarrow h^{-}(\mathbf{X}) \partial_{Z} \mathbf{u}_{0}(\mathbf{x}, 0) \text { and } \tilde{\mathcal{V}}_{1} \equiv 0 .
$$

The value of $\tilde{\mathcal{U}}_{1}$ is chosen as follows: the velocity $\mathbf{u}_{0}$, initially defined for $Z>0$, is naturally defined on $Z<0$ by means of the polynomial extension. The role of $\widetilde{\mathcal{U}}_{1}$ is to compensate the first order error on the physical bottom boundary $y=-\varepsilon^{2} h^{-}(\mathbf{X})$.

Moreover, we will prove that for the solutions $\widetilde{\mathbf{u}}_{1}$ and $\widetilde{v}_{1}$ of $\left(\mathrm{S}^{(1)}\right)$ the limits

$$
\lim _{Y \rightarrow+\infty} \int_{\mathbb{T}^{d}} \widetilde{\mathbf{u}}_{1}(\mathbf{x}, \mathbf{X}, Y) \mathrm{d} \mathbf{X} \text { and } \lim _{Y \rightarrow+\infty} \int_{\mathbb{T}^{d}} \widetilde{v}_{1}(\mathbf{x}, \mathbf{X}, Y) \mathrm{d} \mathbf{X}
$$

exits. They are respectively denoted $\boldsymbol{\alpha}_{1}(\mathbf{x})$ and $\beta_{1}(\mathbf{x})$.

Remark 2.1 Since $\left(\mathbf{u}_{0}, v_{0}, p_{0}\right)$ is the solution of the classical Reynolds equation (see the system $\left(\mathrm{R}^{(0)}\right)$ with $\boldsymbol{A}_{0}=\mathbf{0}, B_{0}=0$ and $C_{0}=0$ ), we easily compute

$$
\widetilde{\mathcal{U}}_{1}: \mathbf{X} \mapsto-h^{-}(\mathbf{X})\left(\frac{h^{+}(\mathbf{x})}{2} \nabla_{\mathbf{x}} p_{0}(\mathbf{x})+\frac{\mathbf{s}}{h^{+}(\mathbf{x})}\right) .
$$

In the same way, we will see that $\beta_{1}=0$ whereas $\boldsymbol{\alpha}_{1} \neq \mathbf{0}$.

Remark 2.2 Notice in particular that the variables $\mathbf{x}$ only plays the role of a parameter in this Stokes problem $\left(\mathrm{S}^{(1)}\right)$. This remark will be common to all the Stokes problems written in this part.

Remark 2.3 Rigorously, the boundary layer problem is time-dependent and should be defined as:

$$
\left\{\begin{aligned}
-\Delta_{\mathbf{X}} \widetilde{\mathbf{u}}_{[T]}-\partial_{Y}^{2} \widetilde{\mathbf{u}}_{[T]}+\nabla_{\mathbf{X}} \widetilde{p}_{[T]} & =\mathbf{0}, & & \text { on } \omega_{b l}(T), \\
-\Delta_{\mathbf{X}} \widetilde{v}_{[T]}-\partial_{Y}^{2} \widetilde{v}_{[T]}+\partial_{Y} \widetilde{p}_{[T]} & =B_{0}, & & \text { on } \omega_{b l}(T), \\
\operatorname{div} \widetilde{\mathbf{u}}_{[T]}+\partial_{Y} \widetilde{v}_{[T]} & =0, & & \text { on } \omega_{b l}(T), \\
\left(\widetilde{\mathbf{u}}_{[T]}, \widetilde{v}_{[T]}\right) & =\left(\widetilde{\mathcal{U}}_{1}(\cdot-\mathbf{s} T), \widetilde{\mathcal{V}}_{1}(\cdot-\mathbf{s} T)\right), & & \text { on } \gamma_{b l}(T) .
\end{aligned}\right.
$$

Notice that the so-called "initial boundary corrector" $\left(\widetilde{\mathbf{u}}_{1}, \widetilde{v}_{1}, \widetilde{p}_{1}\right)$ does not depend on time $T$, unlikely to the boundary corrector solution. But we now argue that the "general boundary corrector" $\left(\widetilde{\mathbf{u}}_{[T]}, \widetilde{v}_{[T]}, \widetilde{p}_{[T]}\right)$ (defined at time $T$ ) can be deduced from the "initial boundary corrector" thanks to the instantaneity of Stokes equations:

$$
\left(\widetilde{\mathbf{u}}_{[T]}, \widetilde{v}_{[T]}, \widetilde{p}_{[T]}\right)(\cdot, \mathbf{X}, Y)=\left(\widetilde{\mathbf{u}}_{1}, \widetilde{v}_{1}, \widetilde{p}_{1}\right)(\cdot, \mathbf{X}-\mathbf{s} T, Y) .
$$

A similar remark can be made on all issues discussed later. 
Remark 2.4 On the same way, the rigorous definition of $\boldsymbol{\alpha}_{1}$ (or of $\beta_{1}$ ), in the construction of the corrector system, should be the following one:

$$
\boldsymbol{\alpha}_{1}(\mathbf{x})=\lim _{Y \rightarrow+\infty} \int_{\mathbb{T}^{d}} \widetilde{\mathbf{u}}_{1}(\mathbf{x}, \mathbf{X}-\mathbf{s} T, Y) \mathrm{d} \mathbf{X}
$$

which, actually, does not depend on $T$ since $\widetilde{\mathbf{u}}_{1}$ is $\mathbf{X}$-periodic (using $\mathbf{X}^{\prime}=\mathbf{X}$ $\mathbf{s} T)$.

Remark 2.5 The behavior of the solution of the Stokes problem is such that

$$
\lim _{Y \rightarrow+\infty} \int_{\mathbb{T}^{d}} \widetilde{v}_{1}(\mathbf{x}, \mathbf{X}, Y) \mathrm{d} \mathbf{X}:=\beta_{1}(\mathbf{x}) .
$$

This limit value is exactly the one that has to be imposed in the definition of the previous Reynolds problem, namely $\left(\mathrm{R}^{(1)}\right)$. At first glance, one may think that problems $\left(\mathrm{R}^{(1)}\right)$ and $\left(\mathrm{S}^{(1)}\right)$ are strongly coupled through the constant $\beta_{1}$ :

- constant $\beta_{1}$ is necessary to define the Reynolds problem;

- constant $\beta_{1}$ results from the behavior of the solution of the Stokes problem, whose data highly depend on the solution of the Reynolds problem.

As will be proven later, not only is each problem well-posed but also - this might be surprising - the two problems are NOT coupled, as $\beta_{1}$ will be proven to be independent from the Stokes problem! See in particular Proposition 3.8 and also subsection 3.3.

Due to the non-zero limit of the integral quantity $\int_{\mathbb{T}^{d}} \widetilde{\mathbf{u}}_{1}(\mathbf{x}, \mathbf{X}, Y) \mathrm{d} \mathbf{X}$ as $Y$ tends to $+\infty$, the contribution of $\widetilde{\mathbf{u}}_{1}$ in the asymptotic development brings an error at the top boundary. This is corrected by the following contribution:

- Second correction at scale $\varepsilon^{1}$ : Reynolds flow in the thin film domain. The functions $\left(\mathbf{u}_{1}, v_{1}, p_{1}\right)$ satisfy the Reynolds problem:

$$
\left(\mathrm{R}^{(1)}\right)\left\{\begin{aligned}
-\partial_{Z}^{2} \mathbf{u}_{1}+\nabla_{\mathbf{x}} p_{1} & =-\boldsymbol{A}_{1}, & & \text { on } \omega_{R}, \\
\partial_{Z} p_{1} & =-B_{1}, & & \text { on } \omega_{R}, \\
\operatorname{div}_{\mathbf{x}} \mathbf{u}_{1}+\partial_{Z} v_{1} & =-C_{1}, & & \text { on } \omega_{R}, \\
\left(\mathbf{u}_{1}, v_{1}\right) & =(\mathbf{0}, 0), & & \text { on } \gamma_{0}, \\
\left(\mathbf{u}_{1}, v_{1}\right) & =\left(-\boldsymbol{\alpha}_{1},-\beta_{2}\right), & & \text { on } \gamma_{+},
\end{aligned}\right.
$$

where the two functions $A_{1}, B_{1}$ and $C_{1}$ only depend on the variable $\mathbf{x}$ and will be precise later (indeed, $\boldsymbol{A}_{1}=\mathbf{0}, B_{1}=0$ and $C_{1}$ is given by equation (18)).

Now, we present the systems satisfied by the corrective terms in the asymptotic expansions: these contributions allow a better description of the initial Stokes flow by increasing the order of the approximation. Notice that the following system highly depends on the previous solutions as source terms. 


\subsection{Higher orders of the asymptotic expansion}

Each order of precision is obtained using a Reynolds flow corresponding to the next order of the thin film assumption; then the corrections due to the roughness patterns have to be taken into account. Notice that the solutions of the previous systems may play the role of source terms in the proposed corrections.

○ Correction at scale $\varepsilon^{j}$ : Stokes flow in the boundary layer.

For $2 \leq j \leq N+1$, the functions $\left(\widetilde{\mathbf{u}}_{j}, \widetilde{v}_{j}, \widetilde{p}_{j}\right)$ satisfy the classical Stokes problem:

$$
\left(\mathrm{S}^{(j)}\right)\left\{\begin{aligned}
-\Delta_{\mathbf{X}} \widetilde{\mathbf{u}}_{j}-\partial_{Y}^{2} \widetilde{\mathbf{u}}_{j}+\nabla_{\mathbf{X}} \widetilde{p}_{j} & =\widetilde{\mathcal{F}}_{j}, & & \text { on } \omega_{b l}(0), \\
-\Delta_{\mathbf{X}} \widetilde{v}_{j}-\partial_{Y}^{2} \widetilde{v}_{j}+\partial_{Y} \widetilde{p}_{j} & =\widetilde{\mathcal{G}}_{j}, & & \text { on } \omega_{b l}(0), \\
\operatorname{div} \mathbf{X} \widetilde{\mathbf{u}}_{j}+\partial_{Y} \widetilde{v}_{j} & =\widetilde{\mathcal{H}}_{j}, & & \text { on } \omega_{b l}(0), \\
\left(\widetilde{\mathbf{u}}_{j}, \widetilde{v}_{j}\right) & =\left(\widetilde{\mathcal{U}}_{j}, \widetilde{\mathcal{V}}_{j}\right), & & \text { on } \gamma_{b l}(0),
\end{aligned}\right.
$$

where the boundary conditions are related to

$$
\begin{gathered}
\widetilde{\mathcal{U}}_{j}: \mathbf{X} \mapsto-\sum_{k=1}^{\left[\frac{j+1}{2}\right]+1} \frac{(-1)^{k} h^{-}(\mathbf{X})^{k}}{k !} \partial_{Z}^{k} \mathbf{u}_{j-k}(\cdot, 0), \\
\tilde{\mathcal{V}}_{j}: \mathbf{X} \mapsto-h^{-}(\mathbf{X}) C_{j-2}(\cdot)+\sum_{k=2}^{\left[\frac{j}{2}\right]+2} \frac{(-1)^{k} h^{-}(\mathbf{X})^{k}}{k !} \operatorname{div}_{\mathbf{X}} \partial_{Z}^{k-1} \mathbf{u}_{j-k-1}(\cdot, 0)
\end{gathered}
$$

The source terms are defined by

$$
\begin{gathered}
\widetilde{\mathcal{F}}_{j}:(\mathbf{X}, Y) \rightarrow \boldsymbol{A}_{j-2}+\left(2 \nabla_{\mathbf{x}} \cdot \nabla_{\mathbf{X}} \widetilde{\mathbf{u}}_{j-2}+\Delta_{\mathbf{x}} \widetilde{\mathbf{u}}_{j-4}-\nabla_{\mathbf{x}} \widetilde{p}_{j-2}\right)(\cdot, \mathbf{X}, Y), \\
\widetilde{\mathcal{G}}_{j}:(\mathbf{X}, Y) \rightarrow B_{j-1}+\left(2 \nabla_{\mathbf{X}} \cdot \nabla_{\mathbf{X}} \widetilde{v}_{j-2}+\Delta_{\mathbf{x}} \widetilde{v}_{j-4}\right)(\cdot, \mathbf{X}, Y) \\
\widetilde{\mathcal{H}}_{j}:(\mathbf{X}, Y) \rightarrow C_{j-2}-\operatorname{div}_{\mathbf{X}} \widetilde{\mathbf{u}}_{j-2}(\cdot, \mathbf{X}, Y) .
\end{gathered}
$$

The value of $\widetilde{\mathcal{U}}_{j}$ and $\widetilde{\mathcal{V}}_{j}$ is chosen as follows: the velocities $\mathbf{u}_{j-k}$ and $v_{j-k}$, $k \geq 1$, initially defined for $Z>0$, are naturally defined on $Z<0$ by means of the polynomial extension. The role of $\widetilde{\mathcal{U}}_{j}$ and $\widetilde{\mathcal{V}}_{j}$ is to compensate the errors on the physical bottom boundary $y=-\varepsilon^{2} h^{-}(\mathbf{X})$.

Moreover, we will prove that, using a good choice for the values $\boldsymbol{A}_{j-2}, B_{j-1}$ and $C_{j-2}$ (see equations $\left.(16)-(18)\right)$, the solutions $\widetilde{\mathbf{u}}_{j}$ and $\widetilde{v}_{j}$ of $\left(\mathrm{S}^{(j)}\right)$ are such that

$$
\lim _{Y \rightarrow+\infty} \int_{\mathbb{T}^{d}} \widetilde{\mathbf{u}}_{j}(\mathbf{x}, \mathbf{X}, Y) \mathrm{d} \mathbf{X} \text { and } \lim _{Y \rightarrow+\infty} \int_{\mathbb{T}^{d}} \widetilde{v}_{j}(\mathbf{x}, \mathbf{X}, Y) \mathrm{d} \mathbf{X}
$$

exist (and will be denoted $\boldsymbol{\alpha}_{j}(\mathbf{x})$ and $\beta_{j}(\mathbf{x})$ respectively).

Remark 2.6 Note that for small values of the integer $j$, the expressions of the source terms are slightly different. In fact, by convention we must read 0 when a term is not defined. For example, $\widetilde{\mathcal{F}}_{2}=\boldsymbol{A}_{0}$ since $\widetilde{\mathbf{u}}_{0}=\mathbf{0}, \widetilde{\mathbf{u}}_{-2}=\mathbf{0}$ and $\widetilde{p}_{0}=0$. 
Remark 2.7 The boundary terms given by $\widetilde{\mathcal{U}}_{j}$ and $\widetilde{\mathcal{V}}_{j}$ come from to the error due to the extension of all the previous interior terms $\mathbf{u}_{k}$ and $v_{k}, k<j$, see their extensions (5) on page 19.

- Main flow at scale $\varepsilon^{j}$ : Reynolds flow in the thin film domain.

For $2 \leq j \leq N$, the functions $\left(\mathbf{u}_{j}, v_{j}, p_{j}\right)$ satisfy the Reynolds problem:

$$
\left(\mathrm{R}^{(j)}\right)\left\{\begin{aligned}
-\partial_{Z}^{2} \mathbf{u}_{j}+\nabla_{\mathbf{x}} p_{j} & =\mathcal{F}_{j}, & & \text { on } \omega_{R} \\
\partial_{Z} p_{j} & =\mathcal{G}_{j}, & & \text { on } \omega_{R}, \\
\operatorname{div}_{\mathbf{x}} \mathbf{u}_{j}+\partial_{Z} v_{j} & =\mathcal{H}_{j}, & & \text { on } \omega_{R}, \\
\left(\mathbf{u}_{j}, v_{j}\right) & =(\mathbf{0}, 0), & & \text { on } \gamma_{0}, \\
\left(\mathbf{u}_{j}, v_{j}\right) & =\left(-\boldsymbol{\alpha}_{j},-\beta_{j+1}\right), & & \text { on } \gamma_{+},
\end{aligned}\right.
$$

with the general source terms

$$
\begin{gathered}
\mathcal{F}_{j}:(\mathbf{x}, Z) \rightarrow-\boldsymbol{A}_{j}(\mathbf{x})+\Delta_{\mathbf{x}} \mathbf{u}_{j-2}(\mathbf{x}, Z), \\
\mathcal{G}_{j}:(\mathbf{x}, Z) \rightarrow-B_{j}(\mathbf{x})+\partial_{Z}^{2} v_{j-2}(\mathbf{x}, Z)+\Delta_{\mathbf{x}} v_{j-4}(\mathbf{x}, Z), \\
\mathcal{H}_{j}:(\mathbf{x}, Z) \rightarrow-C_{j}(\mathbf{x}) .
\end{gathered}
$$

Remark 2.8 As previously, note that for small values of the integer $j$, the expressions of the source terms are slightly different. By convention we must read 0 when a term is not defined. For example $\mathcal{G}_{2}=-B_{2}+\partial_{Z}^{2} v_{0}$ since $v_{-2}=0$.

Remark 2.9 Coefficient $\beta_{j+1}$ should be related to the corrective procedure (although it will be proven to be independent from the corrective procedure) at the next step. More precisely, the behavior at infinity of the solution of the Stokes problem $\left(\mathrm{S}^{(j)}\right)$ is such that

$$
\lim _{Y \rightarrow+\infty} \int_{\mathbb{T}^{d}} \widetilde{v}_{j+1}(\mathbf{x}, \mathbf{X}, Y) \mathrm{d} \mathbf{X}:=\beta_{j+1}(\mathbf{x}) .
$$

This limit value is exactly the one that has to be imposed in the definition of the Reynolds problem $\left(\mathrm{R}^{(j)}\right)$. At first glance, one may think that, by means of construction, problems $\left(\mathrm{R}^{(j+1)}\right)$ and $\left(\mathrm{S}^{(j+1)}\right)$ are strongly coupled through the constant $\beta_{j+1}$. As will be proven later, not only is each problem well-posed but also - this might be surprising - the two problems are NOT coupled, as $\beta_{j+1}$ will be proven to be independent from the Stokes problem! See in particular Proposition 3.8 and also subsection 3.3 (algorithm).

Then, subtracting the asymptotic expansion from the initial solution, we easily find that the remainder should satisfy a Stokes system in the initial domain, with source terms which highly depend on the above solutions, see section 4 . In order to make the previous asymptotic expansion rigorous, we will have to control the remainder. Before entering into the details of the definition and control of the remainder, let us describe the mathematical properties of the Reynolds-type and Stokes-type problems which have been presented in this section. 


\section{Mathematical results related to the different scale problems}

\subsection{Stokes problems: well-posedness and behavior of the solutions}

In this section, we show that the Stokes problems $\left(\mathrm{S}^{(j)}\right)$ are well posed, and we prove that for a suitable choice of the "constants" $\boldsymbol{A}_{j}, B_{j}$ and $C_{j}$, the limits

$$
\lim _{Y \rightarrow+\infty} \int_{\mathbb{T}^{d}} \widetilde{\mathbf{u}}_{j}(\mathbf{x}, \mathbf{X}, Y) \mathrm{d} \mathbf{X} \quad \text { and } \quad \lim _{Y \rightarrow+\infty} \int_{\mathbb{T}^{d}} \widetilde{v}_{j}(\mathbf{x}, \mathbf{X}, Y) \mathrm{d} \mathbf{X},
$$

do exist. We present a result (see Proposition 3.2) whose interest is twofold: i) it allows us to obtain a well-posedness result on the Stokes problems $\left(\mathrm{S}^{(j)}\right)$ by using a lift procedure and classical results on Stokes systems in semi-infinite domains; ii) it allows us to explain how the boundary conditions and the source term in the divergence equation can be translated into boundary conditions on the plane $Y=0$.

Definition 3.1 Let $\widetilde{\mathcal{H}} \in \mathcal{C}\left(\mathbb{T}^{d} \times \mathbb{R}, \mathbb{R}\right), \widetilde{\mathcal{U}} \in \mathcal{C}\left(\mathbb{T}^{d}, \mathbb{R}^{d}\right)$ and $\widetilde{\mathcal{V}} \in \mathcal{C}\left(\mathbb{T}^{d}, \mathbb{R}\right)$ and let us consider $(\widetilde{\mathbf{u}}, \widetilde{v})$ such that

$$
\left\{\begin{aligned}
\operatorname{div}_{\mathbf{X}} \widetilde{\mathbf{u}}+\partial_{Y} \widetilde{v} & =\widetilde{\mathcal{H}}, & & \text { on } \omega_{b l}, \\
(\widetilde{\mathbf{u}}, \widetilde{v}) & =(\widetilde{\mathcal{U}}, \widetilde{\mathcal{V}}), & & \text { on } \gamma_{b l} .
\end{aligned}\right.
$$

Then we define the linear operators

$$
\mathrm{L}_{u}:(\widetilde{\mathcal{H}}, \widetilde{\mathcal{U}}, \widetilde{\mathcal{V}}) \mapsto \int_{\mathbb{T}^{d}} \widetilde{\mathbf{u}}(\mathbf{X}, 0) \mathrm{d} \mathbf{X} \in \mathbb{R}^{d}, \quad \mathrm{~L}_{v}:(\widetilde{\mathcal{H}}, \widetilde{\mathcal{U}}, \widetilde{\mathcal{V}}) \mapsto \int_{\mathbb{T}^{d}} \widetilde{v}(\mathbf{X}, 0) \mathrm{d} \mathbf{X} \in \mathbb{R} .
$$

Remark 3.1 The existence of a solution to equation (3) follows from the fact that for all function $\widetilde{\mathbf{u}}$ on $\omega_{b l}$ such that $\widetilde{\mathbf{u}}=\widetilde{\mathcal{U}}$ on $Y=-h^{-}(\mathbf{X})$, the couple

$$
\left(\widetilde{\mathbf{u}}(\mathbf{X}, Y), \widetilde{\mathcal{V}}(\mathbf{X})+\int_{-h^{-}(\mathbf{X})}^{Y}\left(\widetilde{\mathcal{H}}-\operatorname{div}_{\mathbf{X}} \widetilde{\mathbf{u}}\right)(\mathbf{X}, \zeta) \mathrm{d} \zeta\right)
$$

defines a solution of (3).

We will see that, in practical cases, the velocity imposed at the bottom has a peculiar form. We will use the following proposition.

Proposition 3.2 Let $\mathbf{f} \in \mathcal{C}^{\infty}\left(\mathbb{R}, \mathbb{R}^{d}\right), f \in \mathcal{C}^{\infty}(\mathbb{R}, \mathbb{R}), \widetilde{\mathcal{H}} \in \mathcal{C}\left(\mathbb{T}^{d} \times \mathbb{R}, \mathbb{R}\right)$. Then

$$
\mathrm{L}_{v}\left(\widetilde{\mathcal{H}}, \mathbf{f}\left(h^{-}(\mathbf{X})\right), f\left(h^{-}(\mathbf{X})\right)\right)=-\int_{\{Y<0\}} \widetilde{\mathcal{H}}(\mathbf{X}, Y) \mathrm{d} \mathbf{X} \mathrm{d} Y-\int_{\mathbb{T}^{d}} f\left(h^{-}(\mathbf{X})\right) \mathrm{d} \mathbf{X} .
$$


Proof. We apply the Green's formula :

$$
\begin{aligned}
\int_{\{Y<0\}} \tilde{\mathcal{H}}(\mathbf{X}, Y) \mathrm{d} \mathbf{X} \mathrm{d} Y & =\int_{\{Y<0\}}\left(\operatorname{div}_{\mathbf{X}} \widetilde{\mathbf{u}}+\partial_{Y} \widetilde{v}\right) \\
= & \int_{\mathbb{T}^{d}}\left(\begin{array}{c}
\widetilde{\mathbf{u}}\left(\mathbf{X},-h^{-}(\mathbf{X})\right) \\
\widetilde{v}\left(\mathbf{X},-h^{-}(\mathbf{X})\right)
\end{array}\right) \cdot\left(\begin{array}{c}
-\nabla \mathbf{X} h^{-}(\mathbf{X}) \\
-1
\end{array}\right) \mathrm{d} \mathbf{X} \\
& \quad+\int_{\mathbb{T}^{d}}\left(\begin{array}{c}
\widetilde{\mathbf{u}}(\mathbf{X}, 0) \\
\widetilde{v}(\mathbf{X}, 0)
\end{array}\right) \cdot\left(\begin{array}{c}
\mathbf{0} \\
1
\end{array}\right)(-\mathrm{d} \mathbf{X}) .
\end{aligned}
$$

As $\widetilde{\mathbf{u}}$ and $\widetilde{v}$ have a particular shape on the bottom boundary, we obtain

$$
\begin{array}{r}
\int_{\{Y<0\}} \widetilde{\mathcal{H}}(\mathbf{X}, Y) \mathrm{d} \mathbf{X} \mathrm{d} Y=-\int_{\mathbb{T}^{d}} f\left(h^{-}(\mathbf{X})\right) \mathrm{d} \mathbf{X}-\int_{\gamma_{b l}} \mathbf{f}\left(h^{-}(\mathbf{X})\right) \cdot \nabla_{\mathbf{X}} h^{-}(\mathbf{X}) \mathrm{d} \mathbf{X} \\
-\int_{\mathbb{T}^{d}} \widetilde{v}(\mathbf{X}, 0) \mathrm{d} \mathbf{X} .
\end{array}
$$

By periodicity of function $h^{-}$, we have

$$
\int_{\gamma_{b l}} \mathbf{f}\left(h^{-}(\mathbf{X})\right) \cdot \nabla_{\mathbf{X}} h^{-}(\mathbf{X}) \mathrm{d} \mathbf{X}=\int_{\gamma_{b l}} \nabla_{\mathbf{X}} \mathbf{F}\left(h^{-}(\mathbf{X})\right) \mathrm{d} \mathbf{X}=0,
$$

where $\mathbf{F}$ is a primitive of $\mathbf{f}$. This concludes the proof.

@blacksquare

As a consequence of Proposition 3.2, it is possible to define a lift procedure, so that problem $\left(\mathrm{S}^{(j)}\right)$ reduces to an associated Stokes problem with free-divergence and homogeneous boundary conditions. Well-posedness of such a Stokes problem is well-known (see $[16,17,18,25]$ which provide the functional framework). We now focus on the behavior at infinity of the solution of problem $\left(\mathrm{S}^{(j)}\right)$.

\subsubsection{Initialization step: analysis of problem $\left(\mathrm{S}^{(1)}\right)$}

We properly define the Stokes problem $\left(\mathrm{S}^{(1)}\right)$ introduced on page 10 so that it is well-posed and the behavior of the solution is controlled as $Y \rightarrow+\infty$. More precisely, we prove (see appendix A) the following lemma:

Lemma 3.3 There exists a source term $B_{0}$ (in fact $B_{0}=0$ ) such that system $\left(\mathrm{S}^{(1)}\right)$ admits a solution which takes the following form, for all $(\mathbf{X}, Y) \in$ $\left.\mathbb{T}^{d} \times\right] 0,+\infty[$,

$$
\left\{\begin{array}{l}
\widetilde{\mathbf{u}}_{1}(\mathbf{X}, Y)=\mathrm{L}_{u}\left(0, \widetilde{\mathcal{U}}_{1}, \widetilde{\mathcal{V}}_{1}\right)+\sum_{\mathbf{k} \in \mathbb{Z}^{d} \backslash\{\mathbf{0}\}} \mathbf{P}_{\mathbf{k}}^{(1)}(Y) e^{-2 \pi\|\mathbf{k}\| Y+2 \pi i \mathbf{k} \cdot \mathbf{X}} \\
\widetilde{v}_{1}(\mathbf{X}, Y)=\mathrm{L}_{v}\left(0, \widetilde{\mathcal{U}}_{1}, \widetilde{\mathcal{V}}_{1}\right)+\sum_{\mathbf{k} \in \mathbb{Z}^{d} \backslash\{\mathbf{0}\}} Q_{\mathbf{k}}^{(1)}(Y) e^{-2 \pi\|\mathbf{k}\| Y+2 \pi i \mathbf{k} \cdot \mathbf{X}} \\
\widetilde{p}_{1}(\mathbf{X}, Y)=\sum_{\mathbf{k} \in \mathbb{Z}^{d} \backslash\{\mathbf{0}\}} R_{\mathbf{k}}^{(1)}(Y) e^{-2 \pi\|\mathbf{k}\| Y+2 \pi i \mathbf{k} \cdot \mathbf{X}}
\end{array}\right.
$$

where $\mathbf{P}_{\mathbf{k}}^{(1)}$ and $Q_{\mathbf{k}}^{(1)}$ are affine functions and where $R_{\mathbf{k}}^{(1)}$ are constant. 
It is important to notice that the $\mathbf{X}$-average on $\widetilde{\mathbf{u}}_{1}$ (resp. $v_{1}$ ) does not depend on $Y$. We deduce that its limit when $Y$ tends to $+\infty$, denoted $\boldsymbol{\alpha}_{1}$ (resp. $\beta_{1}$ ), satisfies

$$
\boldsymbol{\alpha}_{1}=\mathrm{L}_{u}\left(0, \tilde{\mathcal{U}}_{1}, \widetilde{\mathcal{V}}_{1}\right) \quad\left(\operatorname{resp} . \beta_{1}=\mathrm{L}_{v}\left(0, \tilde{\mathcal{U}}_{1}, \widetilde{\mathcal{V}}_{1}\right)\right)
$$

As a straightforward consequence, we get the following property:

Corollary 3.4 The solution of $\left(\mathrm{S}^{(1)}\right)$ satisfies:

$$
\begin{aligned}
\left\|\widetilde{\mathbf{u}}_{1}(\mathbf{X}, Y)-\boldsymbol{\alpha}_{1}\right\| & \leq C(\delta) e^{-\delta Y} \quad Y>0, \quad \forall \delta<2 \pi \\
\left|\widetilde{v}_{1}(\mathbf{X}, Y)-\beta_{1}\right| & \leq C(\delta) e^{-\delta Y} \quad Y>0, \quad \forall \delta<2 \pi \\
\left|\widetilde{p}_{1}(\mathbf{X}, Y)\right| & \leq C e^{-2 \pi Y} \quad Y>0,
\end{aligned}
$$

where the constant $C(\delta)$ only depends on $\delta$.

\subsubsection{Induction step: analysis of problem $\left(\mathrm{S}^{(j)}\right)$ for $j \geq 2$}

We show in appendix B the following result about the solution of $\left(\mathrm{S}^{(j)}\right)$ introduced on page 13:

Lemma 3.5 Let $j \geq 2$. There exist source terms $\boldsymbol{A}_{j-2}, B_{j-1}$ and $C_{j-2}$ such that

$$
\int_{\mathbb{T}^{d}} \widetilde{\mathcal{F}}_{j}(\mathbf{X}, \cdot) \mathrm{d} \mathbf{X}=\mathbf{0}, \quad \int_{\mathbb{T}^{d}} \widetilde{\mathcal{G}}_{j}(\mathbf{X}, \cdot) \mathrm{d} \mathbf{X}=0, \quad \int_{\mathbb{T}^{d}} \widetilde{\mathcal{H}}_{j}(\mathbf{X}, \cdot) \mathrm{d} \mathbf{X}=0 .
$$

For such a choice, the solution of $\left(\mathrm{S}^{(j)}\right)$ is written, for all $\left.(\mathbf{X}, Y) \in \mathbb{T}^{d} \times\right] 0,+\infty[$,

$$
\left\{\begin{array}{l}
\widetilde{\mathbf{u}}_{j}(\mathbf{X}, Y)=\mathrm{L}_{u}\left(\widetilde{\mathcal{H}}_{j}, \widetilde{\mathcal{U}}_{j}, \widetilde{\mathcal{V}}_{j}\right)+\sum_{\mathbf{k} \in \mathbb{Z}^{d} \backslash\{\mathbf{0}\}} \mathbf{P}_{\mathbf{k}}^{(j)}(Y) e^{-2 \pi\|\mathbf{k}\| Y+2 \pi i \mathbf{k} \cdot \mathbf{X}} \\
\widetilde{v}_{j}(\mathbf{X}, Y)=\mathrm{L}_{v}\left(\widetilde{\mathcal{H}}_{j}, \widetilde{\mathcal{U}}_{j}, \widetilde{\mathcal{V}}_{j}\right)+\sum_{\mathbf{k} \in \mathbb{Z}^{d} \backslash\{\mathbf{0}\}} Q_{\mathbf{k}}^{(j)}(Y) e^{-2 \pi\|\mathbf{k}\| Y+2 \pi i \mathbf{k} \cdot \mathbf{X}} \\
\widetilde{p}_{j}(\mathbf{X}, Y)=\sum_{\mathbf{k} \in \mathbb{Z}^{d} \backslash\{\mathbf{0}\}} R_{\mathbf{k}}^{(j)}(Y) e^{-2 \pi\|\mathbf{k}\| Y+2 \pi i \mathbf{k} \cdot \mathbf{X}}
\end{array}\right.
$$

where $\mathbf{P}_{\mathbf{k}}^{(j)}, Q_{\mathbf{k}}^{(j)}$ and $R_{\mathbf{k}}^{(j)}$ are polynomial functions.

We deduce that the $\mathbf{X}$-average of $\widetilde{\mathbf{u}}_{j}$ and $\widetilde{v}_{j}$ does not depend on $Y$, so that

$$
\boldsymbol{\alpha}_{j}=\mathrm{L}_{u}\left(\widetilde{\mathcal{H}}_{j}, \widetilde{\mathcal{U}}_{j}, \widetilde{\mathcal{V}}_{j}\right) \text { and } \beta_{j}=\mathrm{L}_{v}\left(\widetilde{\mathcal{H}}_{j}, \widetilde{\mathcal{U}}_{j}, \widetilde{\mathcal{V}}_{j}\right)
$$

As a straightforward consequence, we get the following property:

Corollary 3.6 For all $j \geq 2$, the solution of $\left(\mathrm{S}^{(j)}\right)$ satisfies:

$$
\begin{aligned}
\left\|\widetilde{\mathbf{u}}_{j}(\mathbf{X}, Y)-\boldsymbol{\alpha}_{j}\right\| & \leq C(\delta) e^{-\delta Y} \quad Y>0, \quad \forall \delta<2 \pi, \\
\left|\widetilde{v}_{j}(\mathbf{X}, Y)-\beta_{j}\right| & \leq C(\delta) e^{-\delta Y} \quad Y>0, \quad \forall \delta<2 \pi, \\
\left|\widetilde{p}_{j}(\mathbf{X}, Y)\right| & \leq C(\delta) e^{-\delta Y} \quad Y>0, \quad \forall \delta<2 \pi,
\end{aligned}
$$

where $C(\delta)$ only depends on $\delta$. 


\subsection{Well-posedness of the Reynolds problem}

In this part, we show that the Reynolds-type problems $\left(\mathrm{R}^{(j)}\right)$ are well posed as soon as the "constants" $\boldsymbol{A}_{j}, B_{j}$ and $C_{j}$ are chosen as previously. In particular, due to the fact that $C_{j}=\operatorname{div}_{\mathbf{x}} \boldsymbol{\alpha}_{j}$, system $\left(R^{(j)}\right)$ implies that $\mathbf{u}_{j}+\boldsymbol{\alpha}_{j}, v_{j}+\beta_{j+1}$ and $p_{j}$ satisfy the following Reynolds-type problems on $\omega_{R}$ :

$$
(\mathrm{R})\left\{\begin{aligned}
-\partial_{Z}^{2} \mathbf{u}+\nabla_{\mathbf{x}} p & =\mathcal{F}, & & \text { on } \omega_{R} \\
\partial_{Z} p & =\mathcal{G}, & & \text { on } \omega_{R} \\
\operatorname{div}_{\mathbf{x}} \mathbf{u}+\partial_{Z} v & =0, & & \text { on } \omega_{R} \\
(\mathbf{u}, v) & =\left(\mathcal{U}^{0}, \mathcal{V}^{0}\right), & & \text { on } \gamma_{0} \\
(\mathbf{u}, v) & =(\mathbf{0}, 0), & & \text { on } \gamma_{+} .
\end{aligned}\right.
$$

Here, we assume that the data satisfy some regularity assumptions, i. e. $\mathcal{U}^{0} \in$ $C^{\infty}\left(\mathbb{T}^{d}\right)^{d}, \mathcal{V}^{0} \in C^{\infty}\left(\mathbb{T}^{d}\right), \mathcal{F} \in C^{\infty}\left(\omega_{R}\right)^{d}$ and $\mathcal{G} \in C^{\infty}\left(\omega_{R}\right)$. Moreover, we assume that

$$
\int_{\mathbb{T}^{d}} \mathcal{V}^{0}(\mathbf{x}) \mathrm{d} \mathbf{x}=0
$$

which correspond to a compatibility condition for system $(\mathrm{R})$. In fact we have the following result proven in appendix $\mathrm{C}$ :

Lemma 3.7 Under the compatibility condition (4), problem $(\mathrm{R})$ admits a unique solution $(\mathbf{u}, v, p) \in C^{\infty}\left(\omega_{R}\right)^{d} \times C^{\infty}\left(\omega_{R}\right) \times C^{\infty}\left(\omega_{R}\right) / \mathbb{R}$.

Now let us highlight two crucial properties:

- we first show that $\beta_{j+1}$ only depends on the solution of the Stokes problem $\left(\mathrm{S}^{(j-1)}\right)$, as will be proven in Proposition 3.8;

- as a consequence, we show that assumption (4) is always satisfied for the Reynolds problems $\left(\mathrm{R}^{(j)}\right)$, as will be stated in Remark 3.2

Proposition 3.8 Coefficient $\beta_{j+1}$ which couples problems $\left(\mathrm{R}^{(j)}\right)$ and $\left(\mathrm{S}^{(j+1)}\right)$ only depends on the solution of problems $\left(\mathrm{S}^{(j-1)}\right)$ and $\left(\mathrm{R}^{(k)}\right)$ for $k \leq j-2$ :

$$
\begin{aligned}
\beta_{j+1}(\mathbf{x})= & \operatorname{div}_{\mathbf{x}}\left(\int_{\{Y<0\}} \widetilde{\mathbf{u}}_{j-1}(\mathbf{x}, \mathbf{X}, Y) \mathrm{d} \mathbf{X} \mathrm{d} Y\right) \\
& -\operatorname{div}_{\mathbf{x}}\left(\sum_{k=2}^{\left[\frac{j+1}{2}\right]+2} \frac{(-1)^{k}}{k !}\left(\int_{\mathbb{T}^{d}} h^{-}(\mathbf{X})^{k} \mathrm{~d} \mathbf{X}\right) \partial_{Z}^{k-1} \mathbf{u}_{j-k}(\mathbf{x}, 0)\right) .
\end{aligned}
$$

Proof. We recall that, from the Fourier analysis, $\beta_{j+1}=\mathrm{L}_{v}\left(\widetilde{\mathcal{H}}_{j+1}, \widetilde{\mathcal{U}}_{j+1}, \widetilde{\mathcal{V}}_{j+1}\right)$, where $\widetilde{\mathcal{U}}_{j+1}$ can be viewed as a polynomial function of $h^{-}(\mathbf{X})$ and where $\widetilde{\mathcal{V}}_{j+1}$, which is also a polynomial function of $h^{-}(\mathbf{X})$, takes the following form

$$
\widetilde{\mathcal{V}}_{j+1}(\mathbf{X})=-h^{-}(\mathbf{X}) C_{j-1}(\mathbf{x})+\sum_{k=2}^{\left[\frac{j+1}{2}\right]+2} \frac{(-1)^{k} h^{-}(\mathbf{X})^{k}}{k !} \operatorname{div}_{\mathbf{x}} \partial_{Z}^{k-1} \mathbf{u}_{j-k-1}(\mathbf{x}, 0)
$$


Applying Proposition 3.2, we obtain

$$
\begin{aligned}
\beta_{j+1}(\mathbf{x})=- & \int_{\{Y<0\}} \widetilde{\mathcal{H}}_{j+1}(\mathbf{x}, \mathbf{X}, Y) \mathrm{d} \mathbf{X} \mathrm{d} Y+C_{j-1}(\mathbf{x}) \int_{\mathbb{T}^{d}} h^{-}(\mathbf{X}) \mathrm{d} \mathbf{X} \\
& -\left(\sum_{k=2}^{\left[\frac{j+1}{2}\right]+2} \frac{(-1)^{k} h^{-}(\mathbf{X})^{k}}{k !} \operatorname{div}_{\mathbf{x}} \partial_{Z}^{k-1} \mathbf{u}_{j-k-1}(\mathbf{x}, 0) \mathrm{d} \mathbf{X}\right) .
\end{aligned}
$$

Let us rewrite the right-hand side: first, since $\widetilde{\mathcal{H}}_{j+1}=C_{j-1}-\operatorname{div}_{\mathbf{x}} \widetilde{\mathbf{u}}_{j-1}$ we have

$$
\begin{aligned}
\int_{\{Y<0\}} \widetilde{\mathcal{H}}_{j+1} & (\mathbf{x}, \mathbf{X}, Y) \mathrm{d} \mathbf{X} \mathrm{d} Y \\
& =C_{j-1}(\mathbf{x}) \int_{\{Y<0\}} 1 \mathrm{~d} \mathbf{X} \mathrm{d} Y-\int_{\{Y<0\}} \operatorname{div}_{\mathbf{x}} \widetilde{\mathbf{u}}_{j-1}(\mathbf{x}, \mathbf{X}, Y) \mathrm{d} \mathbf{X} \mathrm{d} Y \\
& =C_{j-1}(\mathbf{x})\left(\int_{\mathbb{T}^{d}} h^{-}(\mathbf{X}) \mathrm{d} \mathbf{X}\right)-\operatorname{div}_{\mathbf{x}}\left(\int_{\{Y<0\}} \widetilde{\mathbf{u}}_{j-1}(\mathbf{x}, \mathbf{X}, Y) \mathrm{d} \mathbf{X} \mathrm{d} Y\right) .
\end{aligned}
$$

Then, the last term in the right-hand side is simply treated by putting the $\operatorname{div}_{\mathbf{x}}$ operator out of the partial sum. We obtain the expression announced for $\beta_{j+1}$.

@blacksquare

Remark 3.2 It is important to notice that for the Reynolds problems $\left(\mathrm{R}^{(j)}\right)$, assumption (4) is always satisfied since $\mathcal{V}^{0}=\beta_{j+1}$. From Proposition 3.8, we deduce that $\beta_{j+1}$ is a $\mathbf{x}$-divergence term which implies, due to the periodicity, that

$$
\int_{\mathbb{T}^{d}} \beta_{j+1}(\mathbf{x}) \mathrm{d} \mathbf{x}=0 .
$$

This corresponds to assumption (4).

From this Remark, the compatibility condition (4) is satisfied for the systems $\left(\mathrm{R}^{(j)}\right)$, we deduce the following result:

Corollary 3.9 For all $j \in \mathbb{N}$, Reynolds-type problem $\left(\mathrm{R}^{(j)}\right)$ admits a unique solution $\left(\mathbf{u}_{j}, v_{j}, p_{j}\right) \in C^{\infty}\left(\omega_{R}\right)^{d} \times C^{\infty}\left(\omega_{R}\right) \times C^{\infty}\left(\omega_{R}\right) / \mathbb{R}$.

As we noted in subsection 2.3 for the first order term $\left(\mathbf{u}_{0}, v_{0}, p_{0}\right)$, we can easily show by induction that the solution $\left(\mathbf{u}_{j}, v_{j}, p_{j}\right)$ of problem $\left(\mathrm{R}^{(j)}\right)$ is polynomial with respect to the variable $Z$. Moreover the degree of these polynomials are given by, for any $n \in \mathbb{N}$, $\operatorname{deg} p_{2 n}=\operatorname{deg} p_{2 n+1}=2 n, \operatorname{deg} \mathbf{u}_{2 n}=\operatorname{deg} \mathbf{u}_{2 n+1}=$ $2 n+2, \operatorname{deg} v_{2 n}=\operatorname{deg} v_{2 n+1}=2 n+3$. It is therefore natural to extend the velocity field $\left(\mathbf{u}_{j}, v_{j}\right)$ for $Z<0$, using its (finite) Taylor development with respect to $Z$. Due to the boundary Dirichlet condition imposed on $\left(\mathbf{u}_{j}, v_{j}\right)$ for $Z=0$, and due to the divergence condition on this velocity (see the problem $\left(\mathrm{R}^{(j)}\right)$ ) we obtain, for all $j \in \mathbb{N} \backslash\{0\}$,

$$
\mathbf{u}_{j}(\cdot, Z)=\sum_{k=1}^{j+2} \partial_{Z}^{k} \mathbf{u}_{j}(\cdot, 0) \frac{Z^{k}}{k !}, v_{j}(\cdot, Z)=-C_{j}-\sum_{k=2}^{j+3} \operatorname{div}_{\mathbf{x}} \partial_{Z}^{k-1} \mathbf{u}_{j}(\cdot, 0) \frac{Z^{k}}{k !}
$$


These are the terms which, measured in $Z=-\varepsilon h^{-}\left(\frac{\mathbf{x}-\mathbf{s} t}{\varepsilon^{2}}\right)$, must be compensated the boundary layer corrector. By Lemmas 3.3, 3.5 and 3.7 (and related corollaries), we have proven that each term of the asymptotic expansion satisfies a well-posed problem. Moreover, we have characterized the behavior of each solution.

\subsection{Algorithm}

In the two previous subsections, we have proven that the intermediate problems, that is Stokes problems $\left(\mathrm{S}^{(j)}\right)$ and Reynolds-type problems $\left(\mathrm{R}^{(j)}\right)$, were all well posed, independently of each other. Clearly, to solve the Stokes problem, you must know some solution of the problem of Reynolds and vice versa. Here, we describe the procedure to really solve all the problems thoroughly.

To evaluate the development up to order $N$ (see the ansatz (2) on page 9), we theoretically add all intermediate profiles: $\left(\mathbf{u}_{0}, v_{0}, p_{0}\right),\left(\widetilde{\mathbf{u}}_{1}, \widetilde{v}_{1}, \widetilde{p}_{1}\right),\left(\mathbf{u}_{1}, v_{1}, p_{1}\right)$, $\left(\widetilde{\mathbf{u}}_{2}, \widetilde{v}_{2}, \widetilde{p}_{2}\right)$ etc. More generally, knowing the terms $\left(\mathbf{u}_{k}, v_{k}, p_{k}\right)$ and $\left(\widetilde{\mathbf{u}}_{k}, \widetilde{v}_{k}, \widetilde{p}_{k}\right)$ for any $k<j$, we compute the terms $\left(\mathbf{u}_{j}, v_{j}, p_{j}\right)$ and $\left(\widetilde{\mathbf{u}}_{j}, \widetilde{v}_{j}, \widetilde{p}_{j}\right)$ as follows.

\section{Initialization:}

(0) Main flow: $\left(\mathbf{u}_{0}, v_{0}, p_{0}\right)$ solves $\left(\mathrm{R}^{(0)}\right)$ with $\boldsymbol{A}_{0}=\mathbf{0}, B_{0}=0, C_{0}=0$, $\beta_{1}=0$.

(1') Corrective Stokes flow: $\left(\widetilde{\mathbf{u}}_{1}, \widetilde{v}_{1}, \widetilde{p}_{1}\right)$ solves $\left(\mathrm{S}^{(1)}\right)$ with

$$
\widetilde{\mathcal{U}}_{1}(\mathbf{X})=h^{-}(\mathbf{X}) \partial_{Z} \mathbf{u}_{0}(\mathbf{x}, 0), \quad \widetilde{\mathcal{V}}_{1} \equiv 0 .
$$

(1") Corrective Reynolds flow: $\left(\mathbf{u}_{1}, v_{1}, p_{1}\right)$ solves $\left(\mathrm{R}^{(1)}\right)$ with

$$
\boldsymbol{\alpha}_{1}=\lim _{Y \rightarrow+\infty} \int_{\mathbb{T}^{d}} \widetilde{\mathbf{u}}_{1}(\cdot, \mathbf{X}, Y) \mathrm{d} \mathbf{X}, \quad \beta_{2}=0, \boldsymbol{A}_{1}=\mathbf{0}, \quad B_{1}=0, C_{1}=\operatorname{div}_{\mathbf{x}} \boldsymbol{\alpha}_{1} .
$$

Iterative procedure: Assume that, for $1 \leq k \leq j-1$,

- problem $\left(\mathrm{S}^{(k)}\right)$, with solution $\left(\widetilde{\mathbf{u}}_{k}, \widetilde{v}_{k}, \widetilde{p}_{k}\right)$, is defined; in particular, the source terms $\left(\widetilde{\mathcal{F}}_{k}, \widetilde{\mathcal{G}}_{k}, \widetilde{\mathcal{H}}_{k}\right)$ and the boundary terms $\left(\widetilde{\mathcal{U}}_{k}, \widetilde{\mathcal{V}}_{k}\right)$ have been defined;

- problem $\left(\mathrm{R}^{(k)}\right)$, with solution $\left.\left(\mathbf{u}_{k}, v_{k}, p_{k}\right)\right)$ is defined; in particular, the source terms $\left(\mathcal{F}_{k}, \mathcal{G}_{k}, \mathcal{H}_{k}\right)$ and the boundary terms $\left(\boldsymbol{\alpha}_{k}, \beta_{k+1}\right)$ have been defined, $i$. e. coefficients $\boldsymbol{A}_{k}, B_{k}, C_{k}$ have been also defined.

(j') Corrective Stokes flow: $\left(\widetilde{\mathbf{u}}_{j}, \widetilde{v}_{j}, \widetilde{p}_{j}\right)$ solves $\left(\mathrm{S}^{(j)}\right)$ with

- the following source terms

$$
\begin{gathered}
\widetilde{\mathcal{F}}_{j}(\mathbf{X}, Y)=\boldsymbol{A}_{j-2}+\left(2 \nabla_{\mathbf{X}} \cdot \nabla_{\mathbf{X}} \widetilde{\mathbf{u}}_{j-2}+\Delta_{\mathbf{x}} \widetilde{\mathbf{u}}_{j-4}-\nabla_{\mathbf{x}} \widetilde{p}_{j-2}\right)(\cdot, \mathbf{X}, Y), \\
\widetilde{\mathcal{G}}_{j}(\mathbf{X}, Y)=B_{j-1}+\left(2 \nabla_{\mathbf{X}} \cdot \nabla_{\mathbf{X}} \widetilde{v}_{j-2}+\Delta_{\mathbf{x}} \widetilde{v}_{j-4}\right)(\cdot, \mathbf{X}, Y), \\
\widetilde{\mathcal{H}}_{j}(\mathbf{X}, Y)=C_{j-2}-\operatorname{div}_{\mathbf{x}} \widetilde{\mathbf{u}}_{j-2}(\cdot, \mathbf{X}, Y) .
\end{gathered}
$$


- the following boundary conditions

$$
\begin{aligned}
& \tilde{\boldsymbol{U}}_{j}(\mathbf{X})=-\sum_{k=1}^{\left[\frac{j+1}{2}\right]+1} \frac{(-1)^{k} h^{-}(\mathbf{X})^{k}}{k !} \partial_{Z}^{k} \mathbf{u}_{j-k}(\mathbf{x}, 0), \\
& \tilde{\mathcal{V}}_{j}(\mathbf{X})=-h^{-}(\mathbf{X}) C_{j-2}(\mathbf{x})+\sum_{k=2}^{\left[\frac{j}{2}\right]+2} \frac{(-1)^{k} h^{-}(\mathbf{X})^{k}}{k !} \operatorname{div}_{\mathbf{x}} \partial_{Z}^{k-1} \mathbf{u}_{j-k-1}(\mathbf{x}, 0) .
\end{aligned}
$$

(j”) Corrective Reynolds flow: $\left(\mathbf{u}_{j}, v_{j}, p_{j}\right)$ solves $\left(\mathrm{R}^{(j)}\right)$ with

- the following boundary values

$$
\begin{aligned}
\boldsymbol{\alpha}_{j}= & \lim _{Y \rightarrow+\infty} \int_{\mathbb{T}^{d}} \widetilde{\mathbf{u}}_{j}(\cdot, \mathbf{X}, Y) \mathrm{d} \mathbf{X} \\
\beta_{j+1}= & \operatorname{div}_{\mathbf{X}}\left(\int_{\{Y<0\}} \widetilde{\mathbf{u}}_{j-1}(\cdot, \mathbf{X}, Y) \mathrm{d} \mathbf{X} \mathrm{d} Y\right) \\
& -\operatorname{div}_{\mathbf{x}}\left(\sum_{k=2}^{\left[\frac{j+1}{2}\right]+2} \frac{(-1)^{k}}{k !}\left(\int_{\mathbb{T}^{d}} h^{-}(\mathbf{X})^{k} \mathrm{~d} \mathbf{X}\right) \partial_{Z}^{k-1} \mathbf{u}_{j-k}(\cdot, 0)\right) .
\end{aligned}
$$

- the following constants: $\boldsymbol{A}_{j}=-\Delta_{\mathbf{x}} \boldsymbol{\alpha}_{j-2}, B_{j}=-\Delta_{\mathbf{x}} \beta_{j-3}, C_{j}=$ $\operatorname{div}_{\mathbf{x}} \boldsymbol{\alpha}_{j}$.

- the following source terms

$$
\begin{gathered}
\mathcal{F}_{j}(\mathbf{x}, Z)=-\boldsymbol{A}_{j}(\mathbf{x})+\Delta_{\mathbf{x}} \mathbf{u}_{j-2}(\mathbf{x}, Z), \\
\mathcal{G}_{j}(\mathbf{x}, Z)=-B_{j}(\mathbf{x})+\partial_{Z}^{2} v_{j-2}(\mathbf{x}, Z)+\Delta_{\mathbf{x}} v_{j-4}(\mathbf{x}, Z), \\
\mathcal{H}_{j}(\mathbf{x})=-C_{j}(\mathbf{x}) .
\end{gathered}
$$

\section{Error analysis}

The error analysis is based on a three-step procedure: $i$ ) first we recall classical estimates related to the Stokes system satisfied by the remainder. At this stage, the estimates do depend on the small parameter $\varepsilon$ through the expression of the source terms and also through the domain $\Omega_{\varepsilon}$ whose measure tends to zero as $\varepsilon$ tends to zero; $i i)$ then we establish estimates which allow us to control the source terms; iii) finally we translate the previous estimates (expressed in a norm which depends on $\varepsilon$ ) into estimates which are relevant with respect to a convergence procedure: the chosen norm preserves the constant states defined in thin domains.

The remainder is defined by the ansatz proposed on equation (2). Using the linearity of the Stokes system, we easily deduce, after some formal computations, that the remainder $\left(\mathcal{R}^{(N)}, \mathcal{S}^{(N)}, \mathcal{Q}^{(N)}\right)$ satisfies a Stokes-type system:

$$
\left(\mathrm{A}_{\varepsilon}\right)\left\{\begin{aligned}
-\Delta_{\mathbf{x}} \mathcal{R}-\partial_{y}^{2} \mathcal{R}+\nabla_{\mathbf{x}} \mathcal{Q} & =\mathcal{F}_{\varepsilon}^{(N)}, & & \text { on } \Omega_{\varepsilon}(t), \\
-\Delta_{\mathbf{x}} \mathcal{S}-\partial_{y}^{2} \mathcal{S}+\partial_{y} \mathcal{Q} & =\mathcal{G}_{\varepsilon}^{(N)}, & & \text { on } \Omega_{\varepsilon}(t), \\
\operatorname{div}_{\mathbf{x}} \mathcal{R}+\partial_{y} \mathcal{S} & =\mathcal{H}_{\varepsilon}^{(N)}, & & \text { on } \Omega_{\varepsilon}(t), \\
(\mathcal{R}, \mathcal{S}) & =\left(\mathcal{U}_{\varepsilon}^{(N)}, \mathcal{V}_{\varepsilon}^{(N)}\right), & & \text { on } \Gamma_{\varepsilon}^{+}, \\
(\mathcal{R}, \mathcal{S}) & =\left(\mathcal{U}_{\varepsilon}^{-(N)}, \mathcal{V}_{\varepsilon}^{-(N)}\right), & & \text { on } \Gamma_{\varepsilon}^{-}(t),
\end{aligned}\right.
$$


where the source terms $\mathcal{F}_{\varepsilon}^{(N)}, \mathcal{G}_{\varepsilon}^{(N)}$ and $\mathcal{H}_{\varepsilon}^{(N)}$ take the following form:

$$
\mathcal{K}_{\varepsilon}^{(N)}(\mathbf{x}, y, t)=\mathcal{K}_{\varepsilon}^{R}\left(\mathbf{x}, \frac{y}{\varepsilon}\right)+\mathcal{K}_{\varepsilon}^{b l}\left(\mathbf{x}, \frac{\mathbf{x}-\mathbf{s} t}{\varepsilon^{2}}, \frac{y}{\varepsilon^{2}}\right) .
$$

with the following precise definitions:

$$
\begin{aligned}
& \mathcal{F}_{\varepsilon}^{R}:=\varepsilon^{N-1}\left(\varepsilon \Delta_{\mathbf{x}} \mathbf{u}_{N}+\Delta_{\mathbf{x}} \mathbf{u}_{N-1}\right), \\
& \mathcal{F}_{\varepsilon}^{b l}:=\varepsilon^{N-2}\left(\varepsilon^{3} \Delta_{\mathbf{x}} \widetilde{\mathbf{u}}_{N+1}+\right. \varepsilon^{2} \Delta_{\mathbf{x}} \widetilde{\mathbf{u}}_{N}+\varepsilon \Delta_{\mathbf{x}} \widetilde{\mathbf{u}}_{N-1}+\Delta_{\mathbf{x}} \widetilde{\mathbf{u}}_{N-2} \\
&\left.+2 \varepsilon \nabla_{\mathbf{x}} \cdot \nabla_{\mathbf{X}} \widetilde{\mathbf{u}}_{N+1}+2 \nabla_{\mathbf{x}} \cdot \nabla_{\mathbf{X}} \widetilde{\mathbf{u}}_{N}-\varepsilon \nabla_{\mathbf{x}} \widetilde{p}_{N+1}-\nabla_{\mathbf{x}} \widetilde{p}_{N}\right), \\
& \mathcal{G}_{\varepsilon}^{R}:=\varepsilon^{N-2}\left(\varepsilon^{3} \Delta_{\mathbf{x}} v_{N}+\varepsilon^{2} \Delta_{\mathbf{x}} v_{N-1}+\varepsilon \Delta_{\mathbf{x}} v_{N-2}+\Delta_{\mathbf{x}} v_{N-3}+\varepsilon \partial_{Z}^{2} v_{N}+\partial_{Z}^{2} v_{N-1}\right), \\
& \mathcal{G}_{\varepsilon}^{b l}:=\varepsilon^{N-2}\left(\varepsilon^{3} \Delta_{\mathbf{x}} \widetilde{v}_{N+1}+\varepsilon^{2} \Delta_{\mathbf{x}} \widetilde{v}_{N}+\varepsilon \Delta_{\mathbf{x}} \widetilde{v}_{N-1}+\Delta_{\mathbf{x}} \widetilde{v}_{N-2}\right. \\
&\left.\quad+2 \varepsilon \nabla_{\mathbf{x}} \cdot \nabla_{\mathbf{X}} \widetilde{v}_{N+1}+2 \nabla_{\mathbf{x}} \cdot \nabla_{\mathbf{X}} \widetilde{v}_{N}\right), \\
& \mathcal{H}_{\varepsilon}^{R}:=0, \quad \\
& \mathcal{H}_{\varepsilon}^{b l}:=-\varepsilon^{N}\left(\varepsilon \operatorname{div}_{\mathbf{x}} \widetilde{\mathbf{u}}_{N+1}+\operatorname{div}_{\mathbf{x}} \widetilde{\mathbf{u}}_{N}\right) .
\end{aligned}
$$

About the boundary condition, using the ansatz at the boundary $\Gamma_{\varepsilon}^{+}$and $\Gamma_{\varepsilon}^{-}$ we get

$$
\begin{aligned}
\mathcal{U}_{\varepsilon}^{(N)}(\mathbf{x}) & =\sum_{j=1}^{N+1} \varepsilon^{j}\left(\boldsymbol{\alpha}_{j}(\mathbf{x})-\widetilde{\mathbf{u}}_{j}\left(\mathbf{x}, \frac{\mathbf{x}}{\varepsilon^{2}}, \frac{h^{+}(\mathbf{x})}{\varepsilon}\right)\right)-\varepsilon^{N+1} \boldsymbol{\alpha}_{N+1}(\mathbf{x}), \\
\mathcal{V}_{\varepsilon}^{(N)}(\mathbf{x}) & =\sum_{j=1}^{N+1} \varepsilon^{j}\left(\beta_{j}(\mathbf{x})-\widetilde{v}_{j}\left(\mathbf{x}, \frac{\mathbf{x}}{\varepsilon^{2}}, \frac{h^{+}(\mathbf{x})}{\varepsilon}\right)\right) \\
\mathcal{U}_{\varepsilon}^{-(N)}(\mathbf{x}) & =\varepsilon^{N+2} \times \text { function }\left(h^{-}\left(\frac{\mathbf{x}-\mathbf{s} t}{\varepsilon}\right), \mathbf{u}_{N}(\mathbf{x}, 0), \ldots, \mathbf{u}_{0}(\mathbf{x}, 0)\right) . \\
\mathcal{V}_{\varepsilon}^{-(N)}(\mathbf{x}) & =\varepsilon^{N+2} \times \text { function }\left(h^{-}\left(\frac{\mathbf{x}-\mathbf{s} t}{\varepsilon}\right), v_{N}(\mathbf{x}, 0), \ldots, v_{0}(\mathbf{x}, 0)\right) .
\end{aligned}
$$

For the sake of simplicity, we do not explicitly give the functions appearing in the boundary terms $\mathcal{U}_{\varepsilon}^{-(N)}$ and $\mathcal{V}_{\varepsilon}^{-(N)}$. They write like the boundary term $\widetilde{\mathcal{U}}_{j}$ and $\widetilde{\mathcal{V}}_{j}$ in the Stokes problem $\left(S^{(j)}\right)$. The existence and uniqueness results of such a problem are well-known (see for example [8]). We will endeavor to obtain estimates of the solution according to the sources terms and to the dependence on $\varepsilon$. By means of construction, as the initial Stokes problem is well-posed and all intermediate problems are also well-posed, we have necessarily

$$
\int_{\Omega_{\varepsilon}} \mathcal{H}_{\varepsilon}^{(N)}=\int_{\Gamma_{\varepsilon}^{+}}\left(\begin{array}{c}
\mathcal{R}^{(N)} \\
\mathcal{S}^{(N)}
\end{array}\right) \cdot \mathbf{n}=\int_{\mathbb{T}^{d}} \mathcal{V}_{\varepsilon}^{(N)}-\int_{\mathbb{T}^{d}} \mathcal{U}_{\varepsilon}^{(N)} \cdot \nabla_{\mathbf{x}} h^{+} .
$$

In the sequel, we will drop the overscripts $(\cdot)^{(N)}$ for the sake of clarity. 


\subsection{Lift procedure}

\subsubsection{Lift velocity at the boundary}

To obtain estimates on the remainder $(\mathcal{R}, \mathcal{S}, \mathcal{Q})$ with respect to $\varepsilon$, we first introduce a explicit velocity field which has the same boundary conditions:

$$
f(\mathbf{x}, y)=\frac{y+\varepsilon^{2} h^{-}\left(\mathbf{x} / \varepsilon^{2}\right)}{\varepsilon h^{+}(\mathbf{x})+\varepsilon^{2} h^{-}\left(\mathbf{x} / \varepsilon^{2}\right)}
$$

and we consider the following velocity field $\left(\widetilde{\mathcal{R}}_{\text {bound }}, \widetilde{\mathcal{S}}_{\text {bound }}\right)$ defined on $\Omega_{\varepsilon}$ by

$$
\begin{aligned}
& \widetilde{\mathcal{R}}_{\text {bound }}(\mathbf{x}, y)=f(\mathbf{x}, y) \mathcal{U}_{\varepsilon}(\mathbf{x})+(1-f(\mathbf{x}, y)) \mathcal{U}_{\varepsilon}^{-}(\mathbf{x}), \\
& \widetilde{\mathcal{S}}_{\text {bound }}(\mathbf{x}, y)=f(\mathbf{x}, y) \mathcal{V}_{\varepsilon}(\mathbf{x})+(1-f(\mathbf{x}, y)) \mathcal{V}_{\varepsilon}^{-}(\mathbf{x})
\end{aligned}
$$

Due to the definition of the function $f$, this vector field satisfies

$$
\left(\widetilde{\mathcal{R}}_{\text {bound }}, \widetilde{\mathcal{S}}_{\text {bound }}\right)=\left(\mathcal{U}_{\varepsilon}, \mathcal{V}_{\varepsilon}\right) \text { on } \Gamma_{\varepsilon}^{+} \quad \text { and } \quad\left(\widetilde{\mathcal{R}}_{\text {bound }}, \widetilde{\mathcal{S}}_{\text {bound }}\right)=\left(\mathcal{U}_{\varepsilon}^{-}, \mathcal{V}_{\varepsilon}^{-}\right) \quad \text { on } \Gamma_{\varepsilon}^{-} \text {. }
$$

\subsubsection{Lift velocity using the Bogovskii formulae}

One of the features of the previous Stokes system is that the divergence of $(\mathcal{R}, \mathcal{S})$ is not equal to zero. A classical method relies on the use of a lifting of the velocity field $(\mathcal{R}, \mathcal{S})$ by introducing a solution $\left(\widetilde{\mathcal{R}}_{\text {div }}, \widetilde{\mathcal{S}}_{\text {div }}\right)$ of the following problem:

$$
\left(\mathrm{A}_{\varepsilon}^{\prime}\right)\left\{\begin{array}{rlrl}
\operatorname{div}_{\mathbf{x}} \widetilde{\boldsymbol{\mathcal { R }}}_{\mathrm{div}}+\partial_{y} \widetilde{\mathcal{S}}_{\mathrm{div}} & =H, & \text { on } \Omega_{\varepsilon}, \\
\left(\widetilde{\mathcal{R}}_{\mathrm{div}}, \widetilde{\mathcal{S}}_{\mathrm{div}}\right) & =(\mathbf{0}, 0), & \text { on } \Gamma_{\varepsilon}^{-}, \\
\left(\widetilde{\mathcal{R}}_{\mathrm{div}}, \widetilde{\mathcal{S}}_{\mathrm{div}}\right) & =(\mathbf{0}, 0), & & \text { on } \Gamma_{\varepsilon}^{+},
\end{array}\right.
$$

where $H=\mathcal{H}_{\varepsilon}-\left(\operatorname{div}_{\mathbf{x}} \widetilde{\mathcal{R}}_{\text {bound }}+\partial_{y} \widetilde{\mathcal{S}}_{\text {bound }}\right)$. An explicit solution of this system exists, corresponding to the Bogovskii formulae (see [6]). The advantage of this formula is to provide precise estimates of the solution. In particular, we have (see for instance [14, p.121]):

Proposition 4.1 (Bogovskii [6]) If $H \in H^{m}\left(\Omega_{\varepsilon}\right), m \geq 0$ has free average then there exists a solution $\left(\widetilde{\mathcal{R}}_{\mathrm{div}}, \widetilde{\mathcal{S}}_{\mathrm{div}}\right) \in H^{m+1}\left(\Omega_{\varepsilon}\right)$ of problem $\left(\mathrm{A}_{\varepsilon}^{\prime}\right)$ such that

$$
\left\|\nabla_{\mathbf{x}, y}\left(\widetilde{\mathcal{R}}_{\mathrm{div}}, \widetilde{\mathcal{S}}_{\mathrm{div}}\right)\right\|_{H^{m}\left(\Omega_{\varepsilon}\right)} \leq \frac{c}{\varepsilon}\|H\|_{H^{m}\left(\Omega_{\varepsilon}\right)}
$$

where the constant $c$ does not depend on $\varepsilon$. Besides, one has also

$$
\left\|\left(\widetilde{\mathcal{R}}_{\text {div }}, \widetilde{\mathcal{S}}_{\text {div }}\right)\right\|_{L^{2}\left(\Omega_{\varepsilon}\right)} \leq c\|H\|_{L^{2}\left(\Omega_{\varepsilon}\right)} .
$$

Remark 4.1 In fact, the constant $c / \varepsilon$ that appears in the right hand side member is explicitly given in [14]. It depends on the number of star-shaped subdomains with respect to some open ball to cover $\Omega_{\varepsilon}$. For the rugous domain $\Omega_{\varepsilon}$, let us focus on the boundary layer $\Omega_{\varepsilon}^{-}(t)$ : the average slope of the roughness patterns is 1 whereas the thickness of the domain is $\varepsilon$ so that the bottom of a 
roughness can be "seen" from a ball of radius $\mathcal{O}(\varepsilon)$. Thus, covering up the domain, whose length is of order 1 , with such balls, we need $\mathcal{O}(1 / \varepsilon)$ balls. Besides, a straightforward use of the Poincaré inequality (note that the domain thickness is of order $\varepsilon$ ) provides the $L^{2}$-bound.

Remark 4.2 Note that the free average condition of the Proposition 4.1 exactly corresponds to the condition (6) satisfied for the Stokes system $\left(\mathrm{A}_{\varepsilon}\right)$.

\subsection{Classical Stokes estimates}

In order to cancel the boundary condition and the divergence of the vector field considered, we define $\overline{\mathcal{R}}=\mathcal{R}-\left(\widetilde{\mathcal{R}}_{\text {bound }}+\widetilde{\mathcal{R}}_{\text {div }}\right)$ and $\overline{\mathcal{S}}=\mathcal{S}-\left(\widetilde{\mathcal{S}}_{\text {bound }}+\widetilde{\mathcal{S}}_{\text {div }}\right)$. We have

$$
\left\{\begin{aligned}
-\Delta_{\mathbf{x}} \overline{\mathcal{R}}-\partial_{y}^{2} \overline{\mathcal{R}}+\nabla_{\mathbf{x}} \mathcal{Q} & =\overline{\mathcal{F}}, & & \text { on } \Omega_{\varepsilon}, \\
-\Delta_{\mathbf{x}} \overline{\mathcal{S}}-\partial_{y}^{2} \overline{\mathcal{S}}+\partial_{y} \mathcal{\mathcal { Q }} & =\overline{\mathcal{G}_{\varepsilon}}, & & \text { on } \Omega_{\varepsilon}, \\
\operatorname{div}_{\mathbf{x}} \overline{\mathcal{R}}+\partial_{y} \overline{\mathcal{S}} & =0, & & \text { on } \Omega_{\varepsilon}, \\
(\overline{\mathcal{R}}, \overline{\mathcal{S}}) & =(\mathbf{0}, 0), & & \text { on } \Gamma_{\varepsilon}^{-}, \\
(\overline{\mathcal{R}}, \overline{\mathcal{S}}) & =(\mathbf{0}, 0), & & \text { on } \Gamma_{\varepsilon}^{+},
\end{aligned}\right.
$$

where $\overline{\mathcal{F}_{\varepsilon}}=\mathcal{F}_{\varepsilon}-\Delta_{\mathbf{x}, y}\left(\widetilde{\mathcal{R}}_{\text {bound }}+\widetilde{\mathcal{R}}_{\text {div }}\right)$ and $\overline{\mathcal{G}_{\varepsilon}}=\mathcal{G}_{\varepsilon}-\Delta_{\text {x }, y}\left(\widetilde{\mathcal{S}}_{\text {bound }}+\widetilde{\mathcal{S}}_{\text {div }}\right)$. We are now able to derive classical estimates:

Proposition 4.2 One has:

i) Estimates in the $L^{2}$-norm:

$$
\|(\overline{\mathcal{R}}, \overline{\mathcal{S}})\|_{L^{2}\left(\Omega_{\varepsilon}\right)} \lesssim \varepsilon^{2}\left\|\left(\overline{\mathcal{F}_{\varepsilon}}, \overline{\mathcal{G}_{\varepsilon}}\right)\right\|_{L^{2}\left(\Omega_{\varepsilon}\right)}, \quad\|\mathcal{Q}\|_{L^{2}\left(\Omega_{\varepsilon}\right)} \lesssim \varepsilon\left\|\left(\overline{\mathcal{F}_{\varepsilon}}, \overline{\mathcal{G}_{\varepsilon}}\right)\right\|_{L^{2}\left(\Omega_{\varepsilon}\right)} .
$$

ii) Estimates in the $H^{1}$-norm:

$$
\|(\overline{\mathcal{R}}, \overline{\mathcal{S}})\|_{H^{1}\left(\Omega_{\varepsilon}\right)} \lesssim \varepsilon\left\|\left(\overline{\mathcal{F}_{\varepsilon}}, \overline{\mathcal{G}_{\varepsilon}}\right)\right\|_{L^{2}\left(\Omega_{\varepsilon}\right)}, \quad\|\mathcal{Q}\|_{H^{1}\left(\Omega_{\varepsilon}\right)} \lesssim\left\|\left(\overline{\mathcal{F}_{\varepsilon}}, \overline{\mathcal{G}_{\varepsilon}}\right)\right\|_{L^{2}\left(\Omega_{\varepsilon}\right)}
$$

Proof. Choosing $\overline{\mathcal{R}}$ as a test function in the first equation, $\overline{\mathcal{S}}$ as test function in the second one and using the free divergence relation, we obtain the following estimate

$$
\begin{array}{r}
\left\|\nabla_{\mathbf{x}} \overline{\mathcal{R}}\right\|_{L^{2}\left(\Omega_{\varepsilon}\right)}^{2}+\left\|\partial_{y} \overline{\mathcal{R}}\right\|_{L^{2}\left(\Omega_{\varepsilon}\right)}^{2}+\left\|\nabla_{\mathbf{x}} \overline{\mathcal{S}}\right\|_{L^{2}\left(\Omega_{\varepsilon}\right)}^{2}+\left\|\partial_{y} \overline{\mathcal{S}}\right\|_{L^{2}\left(\Omega_{\varepsilon}\right)}^{2} \\
\leq\left\|\overline{\mathcal{F}_{\varepsilon}}\right\|_{L^{2}\left(\Omega_{\varepsilon}\right)}\|\overline{\mathcal{R}}\|_{L^{2}\left(\Omega_{\varepsilon}\right)}+\left\|\overline{\mathcal{G}_{\varepsilon}}\right\|_{L^{2}\left(\Omega_{\varepsilon}\right)}\|\overline{\mathcal{S}}\|_{L^{2}\left(\Omega_{\varepsilon}\right)} .
\end{array}
$$

Successively using the Poincaré inequality and the Young inequality $a b \leq \frac{1}{2} a^{2}+$ $\frac{1}{2} b^{2}$ in the right-hand side of the previous inequality, we obtain

$$
\begin{aligned}
\left\|\overline{\mathcal{F}_{\varepsilon}}\right\|_{L^{2}\left(\Omega_{\varepsilon}\right)}\|\overline{\mathcal{R}}\|_{L^{2}\left(\Omega_{\varepsilon}\right)} & \leq c \varepsilon\left\|\overline{\mathcal{F}_{\varepsilon}}\right\|_{L^{2}\left(\Omega_{\varepsilon}\right)}\left\|\partial_{y} \overline{\mathcal{R}}\right\|_{L^{2}\left(\Omega_{\varepsilon}\right)} \\
& \leq \frac{1}{2}\left\|\partial_{y} \overline{\mathcal{R}}\right\|_{L^{2}\left(\Omega_{\varepsilon}\right)}^{2}+\frac{1}{2} c^{2} \varepsilon^{2}\left\|\overline{\mathcal{F}_{\varepsilon}}\right\|_{L^{2}\left(\Omega_{\varepsilon}\right)}^{2},
\end{aligned}
$$


where the constant $c$ does not depend on $\varepsilon$. A similar estimate holds for the other source terms $\left\|\overline{\mathcal{G}_{\varepsilon}}\right\|_{L^{2}\left(\Omega_{\varepsilon}\right)}$ and $\|\overline{\mathcal{S}}\|_{L^{2}\left(\Omega_{\varepsilon}\right)}$. Hence, we successively get (omitting the constants for the sake of simplicity)

$$
\begin{aligned}
&\left\|\nabla_{\mathbf{x}} \overline{\mathcal{R}}\right\|_{L^{2}\left(\Omega_{\varepsilon}\right)}^{2}+\left\|\partial_{y} \overline{\mathcal{R}}\right\|_{L^{2}\left(\Omega_{\varepsilon}\right)}^{2}+\left\|\nabla_{\mathbf{x}} \overline{\mathcal{S}}\right\|_{L^{2}\left(\Omega_{\varepsilon}\right)}^{2}+\left\|\partial_{y} \overline{\mathcal{S}}\right\|_{L^{2}\left(\Omega_{\varepsilon}\right)}^{2} \\
& \lesssim \varepsilon^{2}\left\|\overline{\mathcal{F}_{\varepsilon}}\right\|_{L^{2}\left(\Omega_{\varepsilon}\right)}^{2}+\varepsilon^{2}\left\|\overline{\mathcal{G}_{\varepsilon}}\right\|_{L^{2}\left(\Omega_{\varepsilon}\right)}^{2}
\end{aligned}
$$

Then, using the Poincaré inequality again we obtain

$$
\|\overline{\mathcal{R}}\|_{L^{2}\left(\Omega_{\varepsilon}\right)}^{2}+\|\overline{\mathcal{S}}\|_{L^{2}\left(\Omega_{\varepsilon}\right)}^{2} \lesssim \varepsilon^{4}\left\|\overline{\mathcal{F}_{\varepsilon}}\right\|_{L^{2}\left(\Omega_{\varepsilon}\right)}^{2}+\varepsilon^{4}\left\|\overline{\mathcal{G}_{\varepsilon}}\right\|_{L^{2}\left(\Omega_{\varepsilon}\right)}^{2} .
$$

In the same way, taking respectively $-\Delta_{\mathbf{x}} \overline{\mathcal{R}}-\partial_{y}^{2} \overline{\mathcal{R}}$ and $-\Delta_{\mathbf{x}} \overline{\mathcal{S}}-\partial_{y}^{2} \overline{\mathcal{S}}$ as test functions in the two first equations of the Stokes problem, we get

$$
\left\|\Delta_{\mathbf{x}} \overline{\mathcal{R}}\right\|_{L^{2}\left(\Omega_{\varepsilon}\right)}^{2}+\left\|\partial_{y}^{2} \overline{\mathcal{R}}\right\|_{L^{2}\left(\Omega_{\varepsilon}\right)}^{2}+\left\|\Delta_{\mathbf{x}} \overline{\mathcal{S}}\right\|_{L^{2}\left(\Omega_{\varepsilon}\right)}^{2}+\left\|\partial_{y}^{2} \overline{\mathcal{S}}\right\|_{L^{2}\left(\Omega_{\varepsilon}\right)}^{2} \lesssim\left\|\overline{\mathcal{F}_{\varepsilon}}\right\|_{L^{2}\left(\Omega_{\varepsilon}\right)}^{2}+\left\|\overline{\mathcal{G}_{\varepsilon}}\right\|_{L^{2}\left(\Omega_{\varepsilon}\right)}^{2} .
$$

It is then easy to estimate the pressure:

$$
\left\|\nabla_{\mathbf{x}} \mathcal{Q}\right\|_{L^{2}\left(\Omega_{\varepsilon}\right)}^{2}+\left\|\partial_{y} \mathcal{Q}\right\|_{L^{2}\left(\Omega_{\varepsilon}\right)}^{2} \lesssim\left\|\overline{\mathcal{F}_{\varepsilon}}\right\|_{L^{2}\left(\Omega_{\varepsilon}\right)}^{2}+\left\|\overline{\mathcal{G}_{\varepsilon}}\right\|_{L^{2}\left(\Omega_{\varepsilon}\right)}^{2} .
$$

Using the Poincaré-Wirtinger inequality we obtain

$$
\|\mathcal{Q}\|_{L^{2}\left(\Omega_{\varepsilon}\right)}^{2} \lesssim \varepsilon^{2}\left\|\overline{\mathcal{F}_{\varepsilon}}\right\|_{L^{2}\left(\Omega_{\varepsilon}\right)}^{2}+\varepsilon^{2}\left\|\overline{\mathcal{G}_{\varepsilon}}\right\|_{L^{2}\left(\Omega_{\varepsilon}\right)}^{2} .
$$

All these estimate imply the result announced by the proposition. @blacksquare

Corollary 4.3 In terms of velocities $(\mathcal{R}, \mathcal{S})$, one has:

i) Estimates in the $L^{2}$-norm:

$$
\begin{aligned}
\|(\mathcal{R}, \mathcal{S})\|_{L^{2}\left(\Omega_{\varepsilon}\right) \lesssim} & \varepsilon^{2}\left\|\left(\mathcal{F}_{\varepsilon}, \mathcal{G}_{\varepsilon}\right)\right\|_{L^{2}\left(\Omega_{\varepsilon}\right)}+\varepsilon^{2}\left\|\left(\widetilde{\mathcal{R}}_{\text {bound }}, \widetilde{\mathcal{S}}_{\text {bound }}\right)\right\|_{H^{2}\left(\Omega_{\varepsilon}\right)} \\
& +\varepsilon^{2}\left\|\left(\widetilde{\mathcal{R}}_{\text {div }}, \widetilde{\mathcal{S}}_{\text {div }}\right)\right\|_{H^{2}\left(\Omega_{\varepsilon}\right)}+\left\|\left(\widetilde{\mathcal{R}}_{\text {bound }}, \widetilde{\mathcal{S}}_{\text {bound }}\right)\right\|_{L^{2}\left(\Omega_{\varepsilon}\right)} \\
& +\left\|\left(\widetilde{\mathcal{R}}_{\text {div }}, \widetilde{\mathcal{S}}_{\text {div }}\right)\right\|_{L^{2}\left(\Omega_{\varepsilon}\right)}, \\
\|\mathcal{Q}\|_{L^{2}\left(\Omega_{\varepsilon}\right) \lesssim} & \varepsilon\left\|\left(\mathcal{F}_{\varepsilon}, \mathcal{G}_{\varepsilon}\right)\right\|_{L^{2}\left(\Omega_{\varepsilon}\right)}+\varepsilon\left\|\left(\widetilde{\mathcal{R}}_{\text {bound }}, \widetilde{\mathcal{S}}_{\text {bound }}\right)\right\|_{H^{2}\left(\Omega_{\varepsilon}\right)} \\
& +\varepsilon\left\|\left(\widetilde{\mathcal{R}}_{\text {div }}, \widetilde{\mathcal{S}}_{\text {div }}\right)\right\|_{H^{2}\left(\Omega_{\varepsilon}\right)} .
\end{aligned}
$$

ii) Estimates in the $H^{1}$-norm:

$$
\begin{aligned}
\|(\mathcal{R}, \mathcal{S})\|_{H^{1}\left(\Omega_{\varepsilon}\right) \lesssim} & \varepsilon\left\|\left(\mathcal{F}_{\varepsilon}, \mathcal{G}_{\varepsilon}\right)\right\|_{L^{2}\left(\Omega_{\varepsilon}\right)}+\varepsilon\left\|\left(\widetilde{\mathcal{R}}_{\text {bound }}, \widetilde{\mathcal{S}}_{\text {bound }}\right)\right\|_{H^{2}\left(\Omega_{\varepsilon}\right)} \\
& +\varepsilon\left\|\left(\widetilde{\mathcal{R}}_{\text {div }}, \widetilde{\mathcal{S}}_{\text {div }}\right)\right\|_{H^{2}\left(\Omega_{\varepsilon}\right)}+\left\|\left(\widetilde{\mathcal{R}}_{\text {bound }}, \widetilde{\mathcal{S}}_{\text {bound }}\right)\right\|_{H^{1}\left(\Omega_{\varepsilon}\right)} \\
& +\left\|\left(\widetilde{\mathcal{R}}_{\text {div }}, \widetilde{\mathcal{S}}_{\text {div }}\right)\right\|_{H^{1}\left(\Omega_{\varepsilon}\right)}, \\
\|\mathcal{Q}\|_{H^{1}\left(\Omega_{\varepsilon}\right) \lesssim} & \left\|\left(\mathcal{F}_{\varepsilon}, \mathcal{G}_{\varepsilon}\right)\right\|_{L^{2}\left(\Omega_{\varepsilon}\right)}+\left\|\left(\widetilde{\mathcal{R}}_{\text {bound }}, \widetilde{\mathcal{S}}_{\text {bound }}\right)\right\|_{H^{2}\left(\Omega_{\varepsilon}\right)} \\
& +\left\|\left(\widetilde{\mathcal{R}}_{\text {div }}, \widetilde{\mathcal{S}}_{\text {div }}\right)\right\|_{H^{2}\left(\Omega_{\varepsilon}\right)} .
\end{aligned}
$$




\subsection{Explicit estimates with respect to $\varepsilon$}

\subsubsection{Control of the source terms}

To analyze the terms like $\left\|\mathcal{F}_{\varepsilon}\right\|_{L^{2}\left(\Omega_{\varepsilon}\right)}$ which appear in the estimates given by the Corollary 4.3, we distinguish two kind of terms: those related to a thin film flow, depending on $\mathbf{x}$ and $y / \varepsilon$, and those related to the boundary layer and depending on $\mathbf{x}, \mathbf{x} / \varepsilon^{2}$ and $y / \varepsilon^{2}$. We prove, in appendices $\mathrm{D}$ and $\mathrm{E}$ the following propositions:

Proposition 4.4 Let $f \in \mathcal{C}^{1}\left(\mathbb{T}^{d} \times \omega_{b l}\right)$, such that $\mathbf{X} \mapsto f(\cdot, \mathbf{X}, \cdot)$ is periodic, $f(\cdot, \cdot, Y)=\mathcal{O}\left(e^{-Y}\right)$ for $Y \rightarrow+\infty$ (uniformly w.r.t. the other variables). Let us consider the function $f^{\varepsilon}$ defined by

$$
\forall(\mathbf{x}, y) \in \Omega_{\varepsilon}(t), \quad f^{\varepsilon}(\mathbf{x}, y)=f\left(\mathbf{x}, \frac{\mathbf{x}-\mathbf{s} t}{\varepsilon^{2}}, \frac{y}{\varepsilon^{2}}\right) .
$$

Then we have $\left\|f^{\varepsilon}\right\|_{L^{2}\left(\Omega_{\varepsilon}(t)\right)}^{2} \lesssim \varepsilon^{2}$ and $\left\|f^{\varepsilon}\right\|_{H^{1}\left(\Omega_{\varepsilon}(t)\right)}^{2} \lesssim 1 / \varepsilon^{2}$.

Proposition 4.5 Let $g \in \mathcal{C}^{0}\left(\omega_{R}\right)$ be defined on $\{(\mathbf{x}, Z), Z<0\}$ by a regular extension and let us consider a function $g^{\varepsilon}$ defined by

$$
\forall(\mathbf{x}, y) \in \Omega_{\varepsilon}(t), \quad g^{\varepsilon}(\mathbf{x}, y)=g\left(\mathbf{x}, \frac{y}{\varepsilon}\right) .
$$

Then we have $\left\|g^{\varepsilon}\right\|_{L^{2}\left(\Omega_{\varepsilon}(t)\right)}^{2} \lesssim \varepsilon$.

Recalling the definition of the terms $\mathcal{F}_{\varepsilon}, \mathcal{G}_{\varepsilon}$ and $\mathcal{H}_{\varepsilon}$ (see the subsection 4.1), Propositions 4.4 and 4.5 allow us to derive estimates w.r.t. $\varepsilon$ :

$$
\begin{aligned}
&\left\|\mathcal{F}_{\varepsilon}\right\|_{L^{2}\left(\Omega_{\varepsilon}\right)} \lesssim \varepsilon^{N-1}, \quad\left\|\mathcal{G}_{\varepsilon}\right\|_{L^{2}\left(\Omega_{\varepsilon}\right)} \lesssim \varepsilon^{N-3 / 2}, \\
&\left\|\mathcal{H}_{\varepsilon}\right\|_{L^{2}\left(\Omega_{\varepsilon}\right)} \lesssim \varepsilon^{N+1}, \quad\left\|\mathcal{H}_{\varepsilon}\right\|_{H^{1}\left(\Omega_{\varepsilon}\right)} \lesssim \varepsilon^{N-1}
\end{aligned}
$$

\subsubsection{Boundary lift}

We first use the following estimates about the function $f$ introduced in subsection 4.1.1:

$$
\begin{gathered}
\|f\|_{L^{2}\left(\Omega_{\varepsilon}\right)} \leq \varepsilon^{1 / 2}, \quad\left\|\nabla_{\mathbf{x}} f\right\|_{L^{2}\left(\Omega_{\varepsilon}\right)} \leq \varepsilon^{-1 / 2}, \quad\left\|\partial_{y} f\right\|_{L^{2}\left(\Omega_{\varepsilon}\right)} \leq \varepsilon^{-1 / 2}, \\
\left\|\nabla_{\mathbf{x}} \partial_{y} f\right\|_{L^{2}\left(\Omega_{\varepsilon}\right)} \leq \varepsilon^{-3 / 2}, \quad\left\|\nabla_{\mathbf{x}}^{2} f\right\|_{L^{2}\left(\Omega_{\varepsilon}\right)} \leq \varepsilon^{-5 / 2}, \quad \partial_{y}^{2} f \equiv 0 .
\end{gathered}
$$

We easily deduce the following bounds for the lift velocity at the boundary:

$$
\begin{array}{cc}
\left\|\widetilde{\mathcal{R}}_{\text {bound }}\right\|_{L^{2}\left(\Omega_{\varepsilon}\right)} \leq \varepsilon^{N+3 / 2}, & \left\|\widetilde{\mathcal{S}}_{\text {bound }}\right\|_{L^{2}\left(\Omega_{\varepsilon}\right)} \leq \varepsilon^{N+5 / 2}, \\
\left\|\nabla_{\mathbf{x}} \widetilde{\mathcal{R}}_{\text {bound }}\right\|_{L^{2}\left(\Omega_{\varepsilon}\right)} \leq \varepsilon^{N+1 / 2}, & \left\|\nabla_{\mathbf{x}} \widetilde{\mathcal{S}}_{\text {bound }}\right\|_{L^{2}\left(\Omega_{\varepsilon}\right)} \leq \varepsilon^{N+1 / 2}, \\
\left\|\Delta_{\mathbf{x}} \widetilde{\mathcal{R}}_{\text {bound }}\right\|_{L^{2}\left(\Omega_{\varepsilon}\right)} \leq \varepsilon^{N-3 / 2}, & \left\|\Delta_{\mathbf{x}} \widetilde{\mathcal{S}}_{\text {bound }}\right\|_{L^{2}\left(\Omega_{\varepsilon}\right)} \leq \varepsilon^{N-3 / 2}, \\
\left\|\partial_{y} \widetilde{\mathcal{R}}_{\text {bound }}\right\|_{L^{2}\left(\Omega_{\varepsilon}\right)} \leq \varepsilon^{N+1 / 2}, & \left\|\partial_{y} \widetilde{\mathcal{S}}_{\text {bound }}\right\|_{L^{2}\left(\Omega_{\varepsilon}\right)} \leq \varepsilon^{N+3 / 2}, \\
\partial_{y}^{2} \widetilde{\mathcal{R}}_{\text {bound }} \equiv \mathbf{0}, & \partial_{y}^{2} \widetilde{\mathcal{S}}_{\text {bound }} \equiv 0, \\
\left\|\partial_{y} \nabla_{\mathbf{x}} \widetilde{\mathcal{R}}_{\text {bound }}\right\|_{L^{2}\left(\Omega_{\varepsilon}\right)} \leq \varepsilon^{N-1 / 2}, & \left\|\partial_{y} \nabla_{\mathbf{x}} \widetilde{\mathcal{S}}_{\text {bound }}\right\|_{L^{2}\left(\Omega_{\varepsilon}\right)} \leq \varepsilon^{N-1 / 2}
\end{array}
$$




\subsubsection{Bogovskii lift}

From the estimates for the boundary lift $\left(\widetilde{\mathcal{R}}_{\text {bound }}, \widetilde{\mathcal{S}}_{\text {bound }}\right)$, we can use Proposition 4.1 with a control on the source term $H$ with respect to $\varepsilon$. We obtain

$$
\begin{aligned}
& \left\|\left(\widetilde{\boldsymbol{\mathcal { R }}}_{\text {div }}, \widetilde{\mathcal{S}}_{\text {div }}\right)\right\|_{L^{2}\left(\Omega_{\varepsilon}\right)} \leq \varepsilon^{N+1 / 2}, \\
& \left\|\left(\widetilde{\boldsymbol{\mathcal { R }}}_{\text {div }}, \widetilde{\mathcal{S}}_{\text {div }}\right)\right\|_{H^{1}\left(\Omega_{\varepsilon}\right)} \leq \varepsilon^{N-1 / 2}, \\
& \left\|\left(\widetilde{\boldsymbol{\mathcal { R }}}_{\text {div }}, \widetilde{\mathcal{S}}_{\text {div }}\right)\right\|_{H^{2}\left(\Omega_{\varepsilon}\right)} \leq \varepsilon^{N-3 / 2} .
\end{aligned}
$$

\subsubsection{Estimates}

Coupling the estimates on the source terms (11) and the estimates on the lift (12) and (13) we can rewrite Corollary 4.3 as follows:

Theorem 4.6 (Estimates on the remainder) One has:

$$
\begin{aligned}
&\left\|\left(\mathcal{R}^{(N)}, \mathcal{S}^{(N)}\right)\right\|_{L^{2}\left(\Omega_{\varepsilon}\right)} \lesssim \varepsilon^{N+1 / 2}, \quad\left\|\mathcal{Q}^{(N)}\right\|_{L^{2}\left(\Omega_{\varepsilon}\right)} \lesssim \varepsilon^{N-1 / 2}, \\
&\left\|\left(\boldsymbol{\mathcal { R }}^{(N)}, \mathcal{S}^{(N)}\right)\right\|_{H^{1}\left(\Omega_{\varepsilon}\right)} \lesssim \varepsilon^{N-1 / 2}, \quad\left\|\mathcal{Q}^{(N)}\right\|_{H^{1}\left(\Omega_{\varepsilon}\right)} \lesssim \varepsilon^{N-3 / 2} .
\end{aligned}
$$

This theorem rigorously justifies ansatz (2).

\subsection{Error analysis on adapted spaces}

In this subsection, we translate the previous estimates into similar estimates in which the chosen norm does not depend on the thickness $\varepsilon$. This is motivated by the fact that the $\Omega_{\varepsilon}$-norm of any constant function vanishes as $\varepsilon$ tends to 0 , as the measure of the domain tends to 0 . Thus, estimates have to be expressed in suitable norms that do not depend on $\varepsilon$ and allow us to capture the scale effects in both the rescaled "thin film domain" and the rescaled boundary layer.

Definition 4.7 (Rescaling operator and unfolding operator) Let $\delta$ be $a$ positive integer, and let $\left.(\mathbf{x}, y) \in \mathbb{T}^{d} \times\right] a, b[$.

i) The "rescaling operator"

$$
\begin{array}{ccc}
\mathfrak{R}_{\delta} \quad: \quad L^{2}\left(\mathbb{T}^{d} \times\right] a, b[) & \rightarrow & L^{2}\left(\mathbb{T}^{d} \times\right] a, b[) \\
f & \mapsto & \mathfrak{R}_{\delta}(f),
\end{array}
$$

is defined by $\mathfrak{R}_{\delta}(f)(\cdot, Z):=f(\cdot, \delta Z)$ for all $\left.Z \in\right] a, b[$.

ii) The "unfolding operator"

$$
\begin{array}{ccc}
\mathfrak{U}_{\delta}: \quad L^{2}\left(\mathbb{T}^{d} \times\right] a, b[) & \rightarrow & L^{2}\left(\mathbb{T}^{d} \times \mathbb{T}^{d} \times\right] a, b[) \\
f & \mapsto & \mathfrak{U}_{\delta}(f),
\end{array}
$$

is defined by $\mathfrak{U}_{\delta}(f)(\mathbf{x}, \mathbf{X}, \cdot):=f\left(\delta\left[\frac{\mathbf{x}}{\delta}\right]+\delta \mathbf{X}, \cdot\right)$ for all $(\mathbf{x}, \mathbf{X}) \in \mathbb{T}^{d} \times \mathbb{T}^{d}$, where $[\cdot]$ denotes the integer part in $\mathbb{Z}^{d}$. 
Notice that the so-called "rescaling operator" only rescales the (vertical) last coordinate; the "unfolding operator" only acts on the (horizontal) first variable. The main properties of these operators, from [12], are recalled in appendix F.

The formal development we have introduced requires the separation of the domain $\Omega_{\varepsilon}$ into two "sub-domains": $\omega_{R}$ and $\omega_{b l}$. To take into account the anisotropy of each of these domains, we express the usual $L^{2}\left(\Omega_{\varepsilon}\right)$-norm as follows (the proof of this lemma is given in appendix F).

Lemma 4.8 Let $f \in H^{1}\left(\Omega_{\varepsilon}\right)$. The following estimates hold:

i) Zeroth order derivative:

$$
\|f\|_{L^{2}\left(\Omega_{\varepsilon}\right)}^{2}=\varepsilon\left\|\mathfrak{R}_{\varepsilon}(f)\right\|_{L^{2}\left(\omega_{R}\right)}^{2}+\varepsilon^{2}\left\|\mathfrak{R}_{\varepsilon^{2}} \circ \mathfrak{U}_{\varepsilon^{2}}(f)\right\|_{L^{2}\left(\omega_{b l}\right)}^{2} .
$$

ii) First order derivatives:

$$
\begin{aligned}
& \left\|\nabla_{\mathbf{x}} f\right\|_{L^{2}\left(\Omega_{\varepsilon}\right)}^{2}=\varepsilon^{2}\left\|\nabla_{\mathbf{x}}\left(\mathfrak{R}_{\varepsilon^{2}} \circ \mathfrak{U}_{\varepsilon^{2}}(f)\right)\right\|_{L^{2}\left(\omega_{b l}\right)}^{2} \\
& \quad+\frac{1}{\varepsilon^{2}}\left\|\nabla_{\mathbf{X}}\left(\Re_{\varepsilon^{2}} \circ \mathfrak{U}_{\varepsilon^{2}}(f)\right)\right\|_{L^{2}\left(\omega_{b l}\right)}^{2}+\varepsilon\left\|\nabla_{\mathbf{x}}\left(\mathfrak{R}_{\varepsilon}(f)\right)\right\|_{L^{2}\left(\omega_{R}\right)}^{2}, \\
& \left\|\partial_{y} f\right\|_{L^{2}\left(\Omega_{\varepsilon}\right)}^{2}=\frac{1}{\varepsilon}\left\|\partial_{Z}\left(\Re_{\varepsilon}(f)\right)\right\|_{L^{2}\left(\omega_{R}\right)}^{2}+\frac{1}{\varepsilon^{2}}\left\|\partial_{Y}\left(\Re_{\varepsilon^{2}} \circ \mathfrak{U}_{\varepsilon^{2}}(f)\right)\right\|_{L^{2}\left(\omega_{b l}\right)}^{2} .
\end{aligned}
$$

Now let us define a norm that is adapted to the measure of a function for both the "thin film" approximation and the "roughness boundary layer" aspect:

$$
\llbracket f \rrbracket_{s}^{2}=\left\|\Re_{\varepsilon}(f)\right\|_{H^{s}\left(\omega_{R}\right)}^{2}+\left\|\mathfrak{R}_{\varepsilon^{2}} \circ \mathfrak{U}_{\varepsilon^{2}}(f)\right\|_{H^{s}\left(\omega_{b l}\right)}^{2} .
$$

Unlike the $\Omega_{\varepsilon}$-norms whose drawback is to fail at capturing concentration effects, this norm preserves the constant states independently from the value of $\varepsilon$. Thus, it is a correct way to characterize convergence results in both the thin film region and the boundary layer. We can re-write Theorem 4.6 using the norm $\llbracket \cdot \rrbracket_{s}$ :

Theorem 4.9 (Third estimates on the remainder) One has:
i) L L -estimates: $\llbracket(\mathbf{u}, v)-\left(\mathbf{u}^{(N)}, v^{(N)}\right) \rrbracket_{0} \lesssim \varepsilon^{N-1 / 2}$, $\llbracket p-p^{(N)} \rrbracket_{0} \lesssim \varepsilon^{N-3 / 2}$,
ii) $H^{1}$-estimates: $\llbracket(\mathbf{u}, v)-\left(\mathbf{u}^{(N)}, v^{(N)}\right) \rrbracket_{1} \lesssim \varepsilon^{N-3 / 2}$, $\llbracket p-p^{(N)} \rrbracket_{1} \lesssim \varepsilon^{N-5 / 2}$

\section{Discussion on the different scale effects}

In this section, we discuss two qualitative aspects of the asymptotic expansion. Firstly, we focus on the contribution of the roughness patterns on the thin film approximation. Then we focus on a typical case that is considered in most of the boundary layer studies: the constant cross-section channel, meaning that function $h^{+}$is constant. 


\subsection{Contribution of the rugosities in the thin film approx- imation}

Let us consider the thin film approximation with a flat bottom, i. e. $h^{-}=0$. Then the asymptotic expansion reduces to

$$
\begin{aligned}
& \mathbf{u}_{\triangle}^{(N)}(\mathbf{x}, y, t)=\mathbf{u}_{0}\left(\mathbf{x}, \frac{y}{\varepsilon}\right)+\sum_{j=1}^{N} \varepsilon^{2 j} \mathbf{u}_{2 j}\left(\mathbf{x}, \frac{y}{\varepsilon}\right) \\
& v_{\triangle}^{(N)}(\mathbf{x}, y, t)=\varepsilon v_{0}\left(x, \frac{y}{\varepsilon}\right)+\sum_{j=1}^{N} \varepsilon^{2 j+1} v_{2 j}\left(x, \frac{y}{\varepsilon}\right), \\
& p_{\triangle}^{(N)}(\mathbf{x}, y, t)=\varepsilon^{-2} p_{0}\left(\mathbf{x}, \frac{y}{\varepsilon}\right)+\sum_{j=1}^{N} \varepsilon^{2 j-2} p_{2 j-2}\left(\mathbf{x}, \frac{y}{\varepsilon}\right),
\end{aligned}
$$

meaning that only half of the sequence of Reynolds problems $\left(\mathrm{R}^{(2 j)}\right)$ is considered. Moreover, each Reynolds problem is easy to treat: the pressure obeys an elliptic (Reynolds) equation and the corresponding velocity field can be deduced from the pressure gradient by means of a simple integration, see for instance [27]. When considering the roughness patterns, complexity is greater since we have checked that each Reynolds-type solution of problem $\left(\mathrm{R}^{(2 j)}\right)$ has to be modified by a two-shot correction: $i$ ) a Stokes boundary layer correction and ii) a Reynolds correction.

Besides, it is possible to draw a quantitative study of the convergence of the asymptotic expansion, with or without roughness correction, towards the solution of the full problem. More precisely, suppose that, aiming at evaluating the exact solution defined on $\Omega_{\varepsilon}(t)$, one uses the asymptotic expansion related to the thin film approximation only (i. e. voluntarily omitting the boundary layer corrections); then the error is not controlled by the remainder anymore. If the boundary layer corrections are neglected, the error may be controlled by the lack of precision due to the neglect of the first order boundary layer correction and the related remainder, namely

$$
\begin{aligned}
& \varepsilon \widetilde{\mathbf{u}}_{1}\left(\mathbf{x}, \frac{\mathbf{x}-\mathbf{s} t}{\varepsilon^{2}}, \frac{y}{\varepsilon^{2}}\right)+\varepsilon \mathbf{u}_{1}\left(\mathbf{x}, \frac{y}{\varepsilon}\right)+\mathcal{O}\left(\varepsilon^{2}\right), \\
& \varepsilon \widetilde{v}_{1}\left(\mathbf{x}, \frac{\mathbf{x}-\mathbf{s} t}{\varepsilon^{2}}, \frac{y}{\varepsilon^{2}}\right)+\varepsilon^{2} v_{1}\left(\mathbf{x}, \frac{y}{\varepsilon}\right)+\mathcal{O}\left(\varepsilon^{2}\right), \\
& \varepsilon^{-1} \widetilde{p}_{1}\left(\mathbf{x}, \frac{\mathbf{x}-\mathbf{s} t}{\varepsilon^{2}}, \frac{y}{\varepsilon^{2}}\right)+\varepsilon^{-1} p_{1}\left(\mathbf{x}, \frac{y}{\varepsilon}\right)+\mathcal{O}(1) .
\end{aligned}
$$

Thus the error is controlled by the following estimates (using the results of Propositions 4.4 and 4.5 )

$$
\left\|(\mathbf{u}, v)-\left(\mathbf{u}_{\triangle}^{(N)}, v_{\triangle}^{(N)}\right)\right\|_{L^{2}\left(\Omega_{\varepsilon}\right)} \lesssim \varepsilon^{3 / 2}, \quad\left\|\varepsilon^{2}\left(p-p_{\triangle}^{(N)}\right)\right\|_{L^{2}\left(\Omega_{\varepsilon}\right)} \lesssim \varepsilon^{1 / 2},
$$

whereas the full asymptotic expansion satisfies (see Theorem 4.6):

$$
\left\|(\mathbf{u}, v)-\left(\mathbf{u}^{(N)}, v^{(N)}\right)\right\|_{L^{2}\left(\Omega_{\varepsilon}\right)} \lesssim \varepsilon^{N+1 / 2}, \quad\left\|\varepsilon^{2}\left(p-p^{(N)}\right)\right\|_{L^{2}\left(\Omega_{\varepsilon}\right)} \lesssim \varepsilon^{N+1 / 2} .
$$


Note that, at main order (i. e. $N=1$ ), the truncated velocity field is of or$\operatorname{der} \varepsilon^{3 / 2}$ with or without roughness correction. In contrast, the approximation of the pressure distribution is severely altered by the neglect of the roughness correction; this is a key-point in the framework of lubrication as one may be interested in the control of the load, defined as the $L^{1}$-norm of the pressure in the whole domain.

\subsection{Multiscale coupling effects due to the curvature of the film thickness}

In most of the boundary layer problems dealing with Stokes or Navier-Stokes equations, the typical situation is concerned with a simple geometrical case, as the considered domain is a constant cross-section channel, perturbed by the roughness patterns. More precisely, assuming that

$$
\forall \mathbf{x} \in \mathbb{T}^{d}, \quad h^{+}(\mathbf{x}):=H>0 .
$$

The computation of the solutions becomes much easier than in the full lubrication problem: any Stokes boundary layer problems is such that coefficients do not depend on $\mathbf{x}$ as a parameter, i. e. $\boldsymbol{A}_{j}=\mathbf{0}, B_{j}=0$ and $C_{j}=0$ for all $j \in \mathbb{N}$.

- The main Reynolds problem $\left(\mathrm{R}^{(0)}\right)$ reduces to a simple Couette flow:

$$
\mathbf{u}_{0}(\mathbf{x}, Z)=(1-Z / H) \mathbf{s}, \quad v_{0} \equiv 0, \quad p_{0} \equiv 0 .
$$

- The corrective Stokes problem $\left(\mathrm{S}^{(1)}\right)$ now reads

$$
\left(\mathrm{S}^{(1)}\right)\left\{\begin{aligned}
-\Delta_{\mathbf{X}} \widetilde{\mathbf{u}}_{1}-\partial_{Y}^{2} \widetilde{\mathbf{u}}_{1}+\nabla_{\mathbf{X}} \widetilde{p}_{1} & =\mathbf{0}, & & \text { on } \omega_{b l}(0), \\
-\Delta_{\mathbf{X}} \widetilde{v}_{1}-\partial_{Y}^{2} \widetilde{v}_{1}+\partial_{Y} \widetilde{p}_{1} & =0, & & \text { on } \omega_{b l}(0), \\
\operatorname{div} \mathbf{X} \widetilde{\mathbf{u}}_{1}+\partial_{Y} \widetilde{v}_{1} & =0, & & \text { on } \omega_{b l}(0), \\
\left(\widetilde{\mathbf{u}}_{1}, \widetilde{v}_{1}\right) & =\left(\widetilde{\mathcal{U}}_{1}, 0\right), & & \text { on } \gamma_{b l}(0),
\end{aligned}\right.
$$

where the boundary condition should be read as $\widetilde{\mathcal{U}}_{1}(\mathbf{X})=-\frac{h^{-}(\mathbf{X})}{H} \mathbf{s}$. In the sequel, we will introduce the linear operators $\mathcal{M}_{u}, \mathcal{M}_{v}$ and $\mathcal{M}_{p}$, which only depends on the data $h^{-}$and $H$, such that the solution of the Stokes problem $\left(\mathrm{S}^{(1)}\right)$ reads

$$
\widetilde{\mathbf{u}}_{1}=\mathcal{M}_{u} \mathbf{s}, \quad \widetilde{v}_{1}=\mathcal{M}_{v} \mathbf{s}, \quad \widetilde{p}_{1}=\mathcal{M}_{p} \mathbf{s} .
$$

Remark 5.1 In practice, these operators $\mathcal{M}_{u}, \mathcal{M}_{v}$ and $\mathcal{M}_{p}$ are represented by matrices which are easily obtained by solving d Stokes problems like $\left(\mathrm{S}^{(1)}\right)$ in which we substitute $\mathbf{s}$ by a vectors which form a base for $\mathbb{R}^{d}$.

Using the linearity of this Stokes problem and Proposition 3.2, we deduce that $\boldsymbol{\alpha}_{1}$ and $\beta_{1}$ satisfy $\boldsymbol{\alpha}_{1}=\mathbf{L} \mathbf{s}$ and $\beta_{1}=0$, where $\mathbf{L} \in \mathrm{M}_{d}(\mathbb{R})$ is the matrix of the application $\mathbf{s} \mapsto \frac{-1}{H} \mathrm{~L}_{u}\left(0, h^{-}(\mathbf{X}) \mathbf{s}, 0\right)$. Moreover, we get

$$
\mathbf{L} \mathbf{s}=-\frac{1}{H} \int_{[0,1]^{d}} \mathcal{M}_{u} \mathbf{s}(\mathbf{X}, 0) \mathrm{d} \mathbf{X}
$$


- In that case, the corrective Reynolds problem $\left(\mathrm{R}^{(1)}\right)$ can be written with respect to the unknowns $\mathbf{u}_{1}+\boldsymbol{\alpha}_{1}, v_{1}$ and $p_{1}$. It is similar to the Reynolds problem $\left(\mathrm{R}^{(0)}\right)$, replacing $\mathbf{s}$ with $\boldsymbol{\alpha}_{1}=\mathbf{L} \mathbf{s}$. We obtain

$$
\mathbf{u}_{1}(\mathbf{x}, Z)=-(Z / H) \mathbf{L} \mathbf{s}, \quad v_{1} \equiv 0, \quad p_{1} \equiv 0 .
$$

- In the same way, the corrective Stokes problem $\left(S_{C}^{(2)}\right)$ is similar to the Reynolds problem $\left(S^{(1)}\right)$, replacing $\mathbf{s}$ with $\mathbf{L} \mathbf{s}$. By linearity, we deduce $\widetilde{\mathbf{u}}_{2}=$ $\mathcal{M}_{u} \mathbf{L} \mathbf{s}, \widetilde{v}_{2}=\mathcal{M}_{v} \mathbf{L} \mathbf{s}$, etc. More generally, we obtain, for all $j \in \mathbb{N} \backslash\{0\}$,

$$
\begin{gathered}
\mathbf{u}_{j}(\mathbf{x}, Z)=-(Z / H) \mathbf{L}^{j} \mathbf{s}, \quad v_{j} \equiv 0, \quad p_{j} \equiv 0, \\
\widetilde{\mathbf{u}}_{j}=\mathcal{M}_{u} \mathbf{L}^{j-1} \mathbf{s}, \quad \widetilde{v}_{j}=\mathcal{M}_{v} \mathbf{L}^{j-1} \mathbf{s}, \quad \widetilde{p}_{j}=\mathcal{M}_{p} \mathbf{L}^{j-1} \mathbf{s} .
\end{gathered}
$$

Such analysis implies that, for $N \in \mathbb{N}$, we obtain for instance for the velocity $\mathbf{u}^{(N)}$ :

$$
\mathbf{u}^{(N)}=\mathbf{s}-\left(\frac{\mathcal{Z}}{H}-\varepsilon \mathcal{M}_{u}\right)\left(\left(\mathbf{I d}+\varepsilon \mathbf{L}+\cdots+(\varepsilon \mathbf{L})^{N}\right) \mathbf{s}\right),
$$

where $\mathcal{Z}$ denotes the application $(\mathbf{x}, y, t) \mapsto y / \varepsilon$. We naturally introduce

$$
\begin{gathered}
\mathbf{u}^{(\infty)}=\mathbf{s}-\left(\frac{\mathcal{Z}}{H}-\varepsilon \mathcal{M}_{u}\right)\left((\mathbf{I} \mathbf{d}-\varepsilon \mathbf{L})^{-1} \mathbf{s}\right), \quad v^{(\infty)}=\varepsilon \mathcal{M}_{v}\left((\mathbf{I d}-\varepsilon \mathbf{L})^{-1} \mathbf{s}\right) \\
p^{(\infty)}=\frac{1}{\varepsilon} \mathcal{M}_{p}\left((\mathbf{I} \mathbf{d}-\varepsilon \mathbf{L})^{-1} \mathbf{s}\right) .
\end{gathered}
$$

The function $\left(\mathbf{u}^{(\infty)}, v^{(\infty)}, p^{(\infty)}\right)$ exactly satisfies the Stokes equations in $\Omega_{\varepsilon}$, and the bottom boundary condition corresponding to the initial Stokes problem. Using a Fourier analysis (exactly as in subsection 3.1.1), we show that the boundary condition on the top boundary is satisfied with an error of order $\mathrm{e}^{-H / \varepsilon}$. Following the same method that in section 4, we deduce that, for any Sobolev norms,

$$
\left\|\mathbf{u}-\mathbf{u}^{(\infty)}\right\| \lesssim \mathrm{e}^{-H / \varepsilon}, \quad\left\|v-v^{(\infty)}\right\| \lesssim \mathrm{e}^{-H / \varepsilon}, \quad\left\|p-p^{(\infty)}\right\| \lesssim \mathrm{e}^{-H / \varepsilon} .
$$

Proposition 5.1 If the height $h^{+}$is constant then it suffices to solve d Stokes problems to deduce an approximation with an exponential decreasing error.

\section{A Proof of Lemma 3.3}

Existence of a solution to the Stokes problem $\left(\mathrm{S}^{(1)}\right)$ is usual, see for instance [1, 18]. Moreover, if $\left(\widetilde{\mathbf{u}}_{1}, \widetilde{v}_{1}, \widetilde{p}_{1}\right)$ is a solution of $\left(\mathrm{S}^{(1)}\right)$ satisfying $\nabla \widetilde{\mathbf{u}}_{1}, \nabla \widetilde{v}_{1}, \widetilde{p}_{1}$ $\in L^{2}\left(\mathbb{T}^{d} \times(0,+\infty)\right)$, then it is also a solution of:

$$
\left\{\begin{array}{rlrl}
-\Delta_{\mathbf{X}} \widetilde{\mathbf{u}}_{1}-\partial_{Y}^{2} \widetilde{\mathbf{u}}_{1}+\nabla_{\mathbf{X}} \widetilde{p}_{1} & =\mathbf{0}, & & \text { on } \omega_{b l} \cap\{Y>0\}, \\
-\Delta_{\mathbf{X}} \widetilde{v}_{1}-\partial_{Y}^{2} \widetilde{v}_{1}+\partial_{Y} \widetilde{p}_{1} & =B_{0}, & & \text { on } \omega_{b l} \cap\{Y>0\}, \\
\operatorname{div}_{\mathbf{X}} \widetilde{\mathbf{u}}_{1}+\partial_{Y} \widetilde{v}_{1} & =0, & & \text { on } \omega_{b l} \cap\{Y>0\}, \\
\int_{\mathbb{T}^{d}} \widetilde{\mathbf{u}}_{1}(\mathbf{X}, 0) \mathrm{d} \mathbf{X} & =\mathrm{L}_{u}\left(0, \widetilde{\mathcal{U}}_{1}, \widetilde{\mathcal{V}}_{1}\right), & \\
\int_{\mathbb{T}^{d}} \widetilde{v}_{1}(\mathbf{X}, 0) \mathrm{d} \mathbf{X} & =\mathrm{L}_{v}\left(0, \widetilde{\mathcal{U}}_{1}, \widetilde{\mathcal{V}}_{1}\right) . &
\end{array}\right.
$$


Note that in all the Stokes problems which appear in this paper, the pressures are given up to an additive constant. This constant is chosen here such that

$$
\int_{\mathbb{T}^{d} \times(0,+\infty)} \tilde{p}(\mathbf{X}, Y) \mathrm{d} \mathbf{X} \mathrm{d} Y=0 .
$$

This allows us to pass to the Fourier transform with respect to $\mathbf{X}$ :

$$
\begin{gathered}
\widetilde{\mathbf{u}}_{1}(\mathbf{X}, Y)=\sum_{\mathbf{k} \in \mathbb{Z}^{d}} \widehat{\mathbf{u}_{\mathbf{k}}}(Y) e^{2 \pi i \mathbf{k} \cdot \mathbf{X}}, \quad \widetilde{v}_{1}(\mathbf{X}, Y)=\sum_{\mathbf{k} \in \mathbb{Z}^{d}} \widehat{v_{\mathbf{k}}}(Y) e^{2 \pi i \mathbf{k} \cdot \mathbf{X}}, \\
\widetilde{p}_{1}(\mathbf{X}, Y)=\sum_{\mathbf{k} \in \mathbb{Z}^{d}} \widehat{p_{\mathbf{k}}}(Y) e^{2 \pi i \mathbf{k} \cdot \mathbf{X}} .
\end{gathered}
$$

The previous system on $\left(\widetilde{\mathbf{u}}_{1}, \widetilde{v}_{1}, \widetilde{p}_{1}\right)$ is translated into

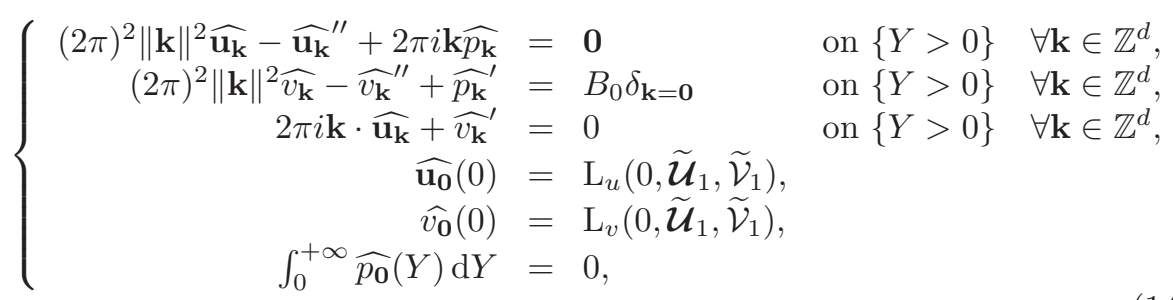

where $\widehat{\mathbf{u}_{\mathbf{k}}},{\widehat{v_{\mathbf{k}}}}^{\prime}$ and $\widehat{p_{\mathbf{k}}}$ belong to $L^{2}(0,+\infty)$. Now we solve the Fourier problem and describe the behavior of the solution of the Stokes problem.

- For $\mathbf{k}=\mathbf{0}$, the system reduces to

$$
\left\{\begin{array}{rllll}
-{\widehat{\mathbf{u}_{\mathbf{0}}}}^{\prime \prime}=\mathbf{0} & \text { with } & \widehat{\mathbf{u}_{\mathbf{0}}}(0)=\mathrm{L}_{u}\left(0, \widetilde{\mathcal{U}}_{1}, \widetilde{\mathcal{V}}_{1}\right) & \text { and } & {\widehat{\mathbf{u}_{0}}}^{\prime} \in L^{2}(0,+\infty), \\
{\widehat{v_{\mathbf{0}}}}^{\prime}=0 & \text { with } & \widehat{v_{\mathbf{0}}}(0)=\mathrm{L}_{v}\left(0, \widetilde{\mathcal{U}}_{1}, \widetilde{\mathcal{V}}_{1}\right) & \text { and } & {\widehat{v_{\mathbf{0}}}}^{\prime} \in L^{2}(0,+\infty), \\
-{\widehat{v_{\mathbf{0}}}}^{\prime \prime}+{\widehat{p_{\mathbf{0}}}}^{\prime}=B_{0} & \text { with } & \int_{0}^{+\infty} \widehat{p_{\mathbf{0}}}(Y) \mathrm{d} Y=0 & \text { and } & \widehat{p_{\mathbf{0}}} \in L^{2}(0,+\infty)
\end{array}\right.
$$

This leads us to the following equalities $\widehat{\mathbf{u}_{\mathbf{0}}}=\mathrm{L}_{u}\left(0, \widetilde{\boldsymbol{U}}_{1}, \widetilde{\mathcal{V}}_{1}\right), \widehat{\boldsymbol{v}_{\mathbf{0}}}=\mathrm{L}_{v}\left(0, \widetilde{\mathcal{U}}_{1}, \widetilde{\mathcal{V}}_{1}\right)$, $\widehat{p_{0}}=0$ with the choice

$$
B_{0}=0 \text {. }
$$

- For $\mathbf{k} \neq \mathbf{0}$, we proceed as follows. The idea is to decompose $\widehat{\mathbf{u}_{\mathbf{k}}}$ as the sum

$$
\widehat{\mathbf{u}_{\mathbf{k}}}=\left(\mathbf{k} \cdot \widehat{\mathbf{u}_{\mathbf{k}}}\right) \mathbf{k}+\left(\mathbf{k}^{\perp} \cdot \widehat{\mathbf{u}_{\mathbf{k}}}\right) \mathbf{k}^{\perp} .
$$

We take the scalar product with $\mathbf{k}^{\perp}$ of the first equation of system (14):

$$
(2 \pi)^{2}\|\mathbf{k}\|^{2}\left(\mathbf{k}^{\perp} \cdot \widehat{\mathbf{u}_{\mathbf{k}}}\right)-\left(\mathbf{k}^{\perp} \cdot \widehat{\mathbf{u}_{\mathbf{k}}}\right)^{\prime \prime}=\mathbf{0} \quad \text { on }\{Y>0\} \quad \forall \mathbf{k} \in \mathbb{Z}^{d} .
$$

Since we have $\mathbf{k}^{\perp} \cdot \widehat{\mathbf{u}_{\mathbf{k}}} \in L^{2}(0,+\infty)$ then it takes the form $a_{\mathbf{k}} e^{-2 \pi\|\mathbf{k}\| Y}$ with $a_{\mathbf{k}} \in \mathbb{R}$. Now, taking the scalar product with $\mathbf{k}$ of the first equation of the system (14), we obtain the pressure with respect to the quantity $\mathbf{k} \cdot \widehat{\mathbf{u}_{\mathbf{k}}}$ :

$$
\widehat{p_{\mathbf{k}}}=2 \pi i\left(\mathbf{k} \cdot \widehat{\mathbf{u}_{\mathbf{k}}}\right)-\frac{i}{2 \pi\|\mathbf{k}\|^{2}}\left(\mathbf{k} \cdot \widehat{\mathbf{u}_{\mathbf{k}}}\right)^{\prime \prime}
$$


Moreover, using the third equation of (14), we express $\mathbf{k} \cdot \widehat{\mathbf{u}_{\mathbf{k}}}$ as a function of $\widehat{v_{\mathbf{k}}}$ :

$$
\mathbf{k} \cdot \widehat{\mathbf{u}_{\mathbf{k}}}=(2 \pi)^{-1} i{\widehat{v_{\mathbf{k}}}}^{\prime},
$$

and then the second equation of the system (14) corresponds to the following homogeneous linear differential equation for the quantity $\widehat{v_{\mathbf{k}}}$ :

$$
\frac{1}{(2 \pi)^{2}\|\mathbf{k}\|^{2}}{\widehat{v_{\mathbf{k}}}}^{\prime \prime \prime \prime}-2{\widehat{v_{\mathbf{k}}}}^{\prime \prime}+(2 \pi)^{2}\|\mathbf{k}\|^{2} \widehat{v_{\mathbf{k}}}=0 .
$$

The solutions of this ODE, which belongs to $L^{2}(0,+\infty)$, take the form

$$
\widehat{v_{\mathbf{k}}}(Y)=\left(c_{\mathbf{k}} Y+d_{\mathbf{k}}\right) e^{-2 \pi\|\mathbf{k}\| Y}, \quad\left(c_{\mathbf{k}}, d_{\mathbf{k}}\right) \in \mathbb{R}^{2} .
$$

By using the expression of $\mathbf{k} \cdot \widehat{\mathbf{u}_{\mathbf{k}}}$ and $\widehat{p_{\mathbf{k}}}$ as a function of $\widehat{v_{\mathbf{k}}}$, we successively get

$$
\begin{aligned}
\mathbf{k} \cdot \widehat{\mathbf{u}_{\mathbf{k}}}(Y) & =(2 \pi)^{-1} i\left(\left(c_{\mathbf{k}}-2 \pi\|\mathbf{k}\| d_{\mathbf{k}}\right)-\|\mathbf{k}\| c_{\mathbf{k}} Y / L\right) e^{-2 \pi\|\mathbf{k}\| Y}, \\
\widehat{p_{\mathbf{k}}}(Y) & =c_{\mathbf{k}} e^{-2 \pi\|\mathbf{k}\| Y} .
\end{aligned}
$$

Finally, we obtain the contribution $\widehat{\mathbf{u}_{\mathbf{k}}}$ using the results for $\mathbf{k} \cdot \widehat{\mathbf{u}_{\mathbf{k}}}$ and $\mathbf{k}^{\perp} \cdot \widehat{\mathbf{u}_{\mathbf{k}}}$. We conclude by defining the following functions:

$$
\begin{aligned}
& \mathbf{P}_{\mathbf{k}}^{(1)}(Y)=(2 \pi)^{-1} i\left(\left(c_{\mathbf{k}}-2 \pi\|\mathbf{k}\| d_{\mathbf{k}}\right)-2 \pi\|\mathbf{k}\| c_{\mathbf{k}} Y\right) \mathbf{k}+a_{\mathbf{k}} \mathbf{k}^{\perp} \\
& Q_{\mathbf{k}}^{(1)}(Y)=c_{\mathbf{k}} Y+d_{\mathbf{k}} \quad \text { and } \quad R_{\mathbf{k}}^{(1)}(Y)=c_{\mathbf{k}} .
\end{aligned}
$$

\section{B Proof of Lemma 3.5}

It is based on the induction.

- Initialization $(j=2)$. Recall that $\widetilde{\mathcal{F}}_{2}=\boldsymbol{A}_{0}, \widetilde{\mathcal{G}}_{2}=B_{1}$ and $\widetilde{\mathcal{H}}_{2}=C_{0}$. In order to ensure that the averages with respect to the variable $\mathbf{X}$ are null, since $\boldsymbol{A}_{0}, B_{1}$ and $C_{0}$ only depend on the variable $\mathbf{x}$, we have to choose

$$
\boldsymbol{A}_{0}=\mathbf{0}, \quad B_{1}=0 \quad \text { and } \quad C_{0}=0 .
$$

Consequently the source terms are null and, as in the proof of Lemma 3.3, we apply a similar procedure. We obtain

$$
\left\{\begin{array}{l}
\widetilde{\mathbf{u}}_{2}(\mathbf{X}, Y)=\mathrm{L}_{u}\left(0, \widetilde{\mathcal{U}}_{2}, \widetilde{\mathcal{V}}_{2}\right)+\sum_{\mathbf{k} \in \mathbb{Z}^{d} \backslash\{\mathbf{0}\}} \mathbf{P}_{\mathbf{k}}^{(2)}(Y) e^{-2 \pi\|\mathbf{k}\| Y+2 \pi i \mathbf{k} \cdot \mathbf{X}} \\
\widetilde{v}_{2}(\mathbf{X}, Y)=\mathrm{L}_{v}\left(0, \widetilde{\mathcal{U}}_{2}, \widetilde{\mathcal{V}}_{2}\right)+\sum_{\mathbf{k} \in \mathbb{Z}^{d} \backslash\{\mathbf{0}\}} Q_{\mathbf{k}}^{(2)}(Y) e^{-2 \pi\|\mathbf{k}\| Y+2 \pi i \mathbf{k} \cdot \mathbf{X}} \\
\widetilde{p}_{2}(\mathbf{X}, Y)=\sum_{\mathbf{k} \in \mathbb{Z}^{d} \backslash\{\mathbf{0}\}} R_{\mathbf{k}}^{(2)}(Y) e^{-2 \pi\|\mathbf{k}\| Y+2 \pi i \mathbf{k} \cdot \mathbf{X}}
\end{array}\right.
$$

where $\mathbf{P}_{\mathbf{k}}^{(2)}, Q_{\mathbf{k}}^{(2)}$ and $R_{\mathbf{k}}^{(2)}$ are affine functions.

- Induction. Let $j \geq 2$ and suppose that Lemma 3.5 holds for any index $k<j$ and let us prove that it still holds for $k=j$. 
First, we have to show that it is possible to choose $\boldsymbol{A}_{j-2}, B_{j-1}$ and $C_{j-2}$ (which do not depend on the Stokes variables $(\mathbf{X}, Y)$ ) such that the source terms are free average with respect to $\mathbf{X}$. Let us recall that

$$
\widetilde{\mathcal{F}}_{j}(\mathbf{X}, Y)=\boldsymbol{A}_{j-2}+\left(2 \nabla_{\mathbf{x}} \cdot \nabla_{\mathbf{X}} \widetilde{\mathbf{u}}_{j-2}+\Delta_{\mathbf{x}} \widetilde{\mathbf{u}}_{j-4}-\nabla_{\mathbf{x}} \widetilde{p}_{j-2}\right)(\cdot, \mathbf{X}, Y) .
$$

Since $\widetilde{\mathbf{u}}_{j-2}$ is $\mathbf{X}$-periodic, and since the $\mathbf{X}$-average of $\widetilde{p}_{j-2}$ is zero by induction assumption, it is sufficient to impose $\boldsymbol{A}_{j-2}=-\Delta_{\mathbf{x}}\left(\int_{\mathbb{T}^{d}} \widetilde{\mathbf{u}}_{j-4}(\cdot, \mathbf{X}, Y) \mathrm{d} \mathbf{X}\right)$, that is

$$
\boldsymbol{A}_{j-2}=-\Delta_{\mathbf{x}} \boldsymbol{\alpha}_{j-4}
$$

It is important to notice that $\boldsymbol{A}_{j-2}$ does not depend on $Y$. In the same way, using the definition $\widetilde{\mathcal{G}}_{j}(\mathbf{X}, Y)=B_{j-1}+\left(2 \nabla_{\mathbf{x}} \cdot \nabla_{\mathbf{X}} \widetilde{v}_{j-2}+\Delta_{\mathbf{x}} \widetilde{v}_{j-4}\right)(\cdot, \mathbf{X}, Y)$, we impose

$$
B_{j-1}=-\Delta_{\mathbf{x}} \beta_{j-4} .
$$

Finally, since we have $\widetilde{\mathcal{H}}_{j}(\mathbf{X}, Y)=C_{j-2}-\operatorname{div}_{\mathbf{x}} \widetilde{\mathbf{u}}_{j-2}(\cdot, \mathbf{X}, Y)$, we impose

$$
C_{j-2}=\operatorname{div}_{\mathbf{x}} \boldsymbol{\alpha}_{j-2} .
$$

For such choices for $\boldsymbol{A}_{j-2}, B_{j-1}$ and $C_{j-2}$, the source terms $\widetilde{\mathcal{F}}_{j}, \widetilde{\mathcal{G}}_{j}$ and $\widetilde{\mathcal{H}}_{j}$ are periodic and free-average with respect to the $\mathbf{X}$ variable. Moreover, thanks to the induction assumption, they take the following form

$$
\begin{aligned}
& \widetilde{\mathcal{F}}_{j}(\mathbf{X}, Y)=\sum_{\mathbf{k} \in \mathbb{Z}^{d} \backslash\{\mathbf{0}\}} \widetilde{\mathbf{P}_{\mathbf{k}}}(Y) e^{-2 \pi\|\mathbf{k}\| Y+2 \pi i \mathbf{k} \cdot \mathbf{X}}, \\
& \widetilde{\mathcal{G}}_{j}(\mathbf{X}, Y)=\sum_{\mathbf{k} \in \mathbb{Z}^{d} \backslash\{\mathbf{0}\}} \widetilde{Q_{k}}(Y) e^{-2 \pi\|\mathbf{k}\| Y+2 \pi i \mathbf{k} \cdot \mathbf{X}}, \\
& \widetilde{\mathcal{H}}_{j}(\mathbf{X}, Y)=\sum_{\mathbf{k} \in \mathbb{Z}^{d} \backslash\{\mathbf{0}\}} \widetilde{R_{k}}(Y) e^{-2 \pi\|\mathbf{k}\| Y+2 \pi i \mathbf{k} \cdot \mathbf{X}},
\end{aligned}
$$

where $\widetilde{\mathbf{P}_{\mathbf{k}}}, \widetilde{Q_{k}}$ and $\widetilde{R_{k}}$ are polynomial. Using the Fourier transform of the system $\left(\mathrm{S}^{(j)}\right)$, we deduce an equivalent system on the Fourier coefficients $\left(\widehat{\mathbf{u}_{\mathbf{k}}}, \widehat{v_{\mathbf{k}}}, \widehat{p_{\mathbf{k}}}\right)$ :

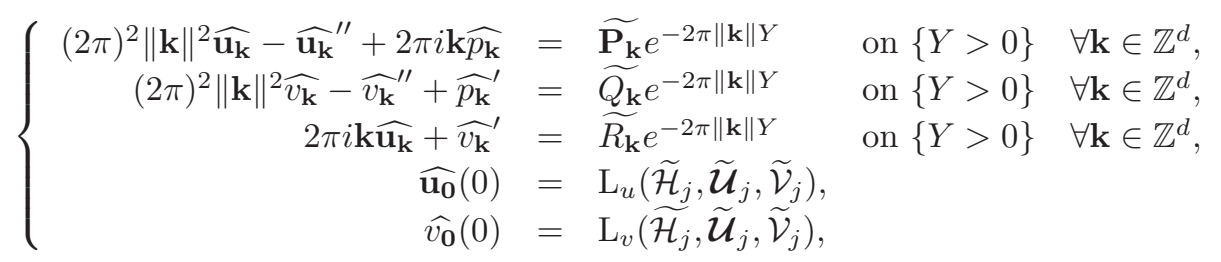

where ${\widehat{\mathbf{u}_{\mathbf{k}}}}^{\prime},{\widehat{v_{\mathbf{k}}}}^{\prime}$ and $\widehat{p_{\mathbf{k}}}$ belong to $L^{2}(0,+\infty)$ and where $\int_{0}^{+\infty} \widehat{p_{\mathbf{0}}}(Y) \mathrm{d} Y=0$.

- For $\mathbf{k}=\mathbf{0}$, since $\widetilde{\mathbf{P}_{\mathbf{0}}}=\mathbf{0}, \widetilde{Q_{\mathbf{0}}}=0$ and $\widetilde{R_{\mathbf{0}}}=0$, we deduce that (see the proof of Lemma 3.5 for the same kind of calculations):

$$
\widehat{\mathbf{u}_{\mathbf{0}}}=\mathrm{L}_{u}\left(\widetilde{\mathcal{H}}_{j}, \widetilde{\mathcal{U}}_{j}, \widetilde{\mathcal{V}}_{j}\right), \quad \widehat{v_{\mathbf{0}}}=\mathrm{L}_{v}\left(\widetilde{\mathcal{H}}_{j}, \widetilde{\mathcal{U}}_{j}, \widetilde{\mathcal{V}}_{j}\right) \quad \text { and } \quad \widehat{p_{0}}=0 .
$$

- For $\mathbf{k} \neq \mathbf{0}$, using the same method as previously (see the proof of Lemma 3.5), we first obtain a linear differential equation on the function defined by $f(Y)=$ $\mathbf{k}^{\perp} \cdot \widehat{\mathbf{u}_{\mathbf{k}}}(Y)$ :

$$
(2 \pi)^{2}\|\mathbf{k}\|^{2} f(Y)-f^{\prime \prime}(Y)=\mathbf{k}^{\perp} \cdot \widetilde{\mathbf{P}_{\mathbf{k}}} e^{-2 \pi\|\mathbf{k}\| Y} .
$$


Since $\widetilde{\mathbf{P}_{\mathbf{k}}}$ is a polynom, the solutions are written $\mathbf{k}^{\perp} \cdot \widehat{\mathbf{u}_{\mathbf{k}}}(Y)=\mathcal{P}_{\mathbf{k}}(Y) e^{-2 \pi\|\mathbf{k}\| Y}$, where $\mathcal{P}_{\mathbf{k}}$ is a polynom. Next we obtain

$$
\begin{aligned}
\widehat{p_{\mathbf{k}}} & =2 \pi i\left(\mathbf{k} \cdot \widehat{\mathbf{u}_{\mathbf{k}}}\right)-\frac{i}{2 \pi\|\mathbf{k}\|^{2}}\left(\mathbf{k} \cdot \widehat{\mathbf{u}_{\mathbf{k}}}\right)^{\prime \prime}-\frac{i}{2 \pi\|\mathbf{k}\|^{2}}\left(\mathbf{k} \cdot \widetilde{\mathbf{P}_{\mathbf{k}}}\right) e^{-2 \pi\|\mathbf{k}\| Y}, \\
\mathbf{k} \cdot \widehat{\mathbf{u}_{\mathbf{k}}} & =\frac{i}{2 \pi}\left(\widehat{v_{\mathbf{k}}}-\widetilde{R_{\mathbf{k}}} e^{-2 \pi\|\mathbf{k}\| Y}\right) .
\end{aligned}
$$

Moreover $\widehat{v_{\mathbf{k}}}$ satisfies the non-homogeneous linear differential equation

$$
\begin{aligned}
\frac{1}{(2 \pi)^{2}\|\mathbf{k}\|^{2}}{\widehat{v_{\mathbf{k}}}}^{\prime \prime \prime \prime}-2{\widehat{v_{\mathbf{k}}}}^{\prime \prime}+(2 \pi)^{2}\|\mathbf{k}\|^{2} \widehat{v_{\mathbf{k}}} \\
=\left(\widetilde{Q_{\mathbf{k}}}+\frac{i}{2 \pi\|\mathbf{k}\|^{2}}\left(\mathbf{k} \cdot \widetilde{\mathbf{P}_{\mathbf{k}}}\right)-\frac{i}{\|\mathbf{k}\|}\left(\mathbf{k} \cdot \widetilde{\mathbf{P}_{\mathbf{k}}}\right)\right) e^{-2 \pi\|\mathbf{k}\| Y} .
\end{aligned}
$$

The solutions of this ODE are the sum of the solution of the homogeneous equation (solved before) and a particular solution which, due to the form of the right-hand side, can be obtained as a product between a polynomial function and $e^{-2 \pi\|\mathbf{k}\| Y}$. From such an expression for $\widehat{v_{\mathbf{k}}}$ the proof is concluded.

\section{Proof of Lemma 3.7}

To study the Reynolds system (R), we use some algebraic transformations:

- Integrating the pressure equation gives

$$
p(\cdot, Z)=\bar{p}+\int_{0}^{Z} \mathcal{G}(\cdot, \zeta) \mathrm{d} \zeta
$$

where $\mathbf{x} \mapsto \bar{p}(\mathbf{x})$ is a function to be determined.

- Then, putting the above equality into the $(\mathbf{u}, p)$ relationship gives

$$
-\partial_{Z}^{2} \mathbf{u}+\nabla_{\mathbf{x}} \bar{p}=\mathcal{F}-\int_{0}^{Z} \nabla_{\mathbf{x}} \mathcal{G}(\cdot, \mu) \mathrm{d} \mu,
$$

which we integrate twice in the $Z$-variable to obtain $\mathbf{u}$. The divergence equation provides an expression of $v$ (by a simple integration).

- Integrating between 0 and $h^{+}$the divergence equation of $(\mathrm{R})$, we get

$$
\begin{aligned}
\operatorname{div}_{\mathbf{x}}\left(\frac{h^{+3}}{12} \nabla_{\mathbf{x}} \bar{p}\right)= & \operatorname{div}_{\mathbf{x}}\left(\frac{h^{+}}{2} \mathcal{U}^{0}\right)-\mathcal{V}^{0} \\
& +\operatorname{div}_{\mathbf{x}}\left(\int_{0}^{h^{+}} \int_{0}^{y} \int_{0}^{\eta}\left\{\mathcal{F}(\cdot, \zeta)-\int_{0}^{\zeta} \nabla_{\mathbf{x}} \mathcal{G}(\cdot, \mu) \mathrm{d} \mu\right\} \mathrm{d} \zeta \mathrm{d} \eta \mathrm{d} y\right) \\
& -\operatorname{div}_{\mathbf{x}}\left(\frac{h^{+}}{2} \int_{0}^{h^{+}} \int_{0}^{\eta}\left\{\mathcal{F}(\cdot, \zeta)-\int_{0}^{\zeta} \nabla_{\mathbf{x}} \mathcal{G}(\cdot, \mu) \mathrm{d} \mu\right\} \mathrm{d} \zeta \mathrm{d} \eta\right)
\end{aligned}
$$

Obviously, equation (20) with assumption (4) can be written as

$$
\operatorname{div}_{\mathbf{x}}\left(\mathcal{A} \nabla_{\mathbf{x}} \bar{p}\right)=\operatorname{div}_{\mathbf{x}} \mathcal{B}-\mathcal{C} \quad \text { with } \quad \int_{\mathbb{T}^{d}} \mathcal{C}=0
$$


where the coefficients $\mathcal{A}=\frac{1}{12} h^{+^{3}}, \mathcal{B}=\mathcal{B}\left(h^{+}, \mathcal{F}, \mathcal{G}, \mathcal{U}^{0}\right)$ and $\mathcal{C}=\mathcal{V}^{0}$ are smooth and depend on all the data. Existence and uniqueness of a solution $\bar{p} \in H^{1}\left(\mathbb{T}^{d}\right) / \mathbb{R}$ immediately follow from the Lax-Milgram theorem. From the regularity of the data, $\bar{p} \in C^{\infty}\left(\mathbb{T}^{d}\right) / \mathbb{R}$; then the regularity of $\mathbf{u}$ and $v$ follows by means of integration.

\section{Proof of Proposition 4.4}

Expressing the $L^{2}$-norm of function $f^{\varepsilon}$, we have

$$
\left\|f^{\varepsilon}\right\|_{L^{2}\left(\Omega_{\varepsilon}(t)\right)}^{2}=\int_{\mathbb{T}^{d}}\left(\int_{-\varepsilon^{2} h^{-}\left((\mathbf{x}-\mathbf{s} t) / \varepsilon^{2}\right)}^{\varepsilon h^{+}(\mathbf{x})}\left|f\left(\mathbf{x}, \frac{\mathbf{x}-\mathbf{s} t}{\varepsilon^{2}}, \frac{y}{\varepsilon^{2}}\right)\right|^{2} \mathrm{~d} y\right) \mathrm{d} \mathbf{x} .
$$

Using the change of variable $Y=y / \varepsilon^{2}$, we get

$$
\begin{aligned}
\left\|f^{\varepsilon}\right\|_{L^{2}\left(\Omega_{\varepsilon}(t)\right)}^{2} & =\varepsilon^{2} \int_{\mathbb{T}^{d}}\left(\int_{-h^{-}\left((\mathbf{x}-\mathbf{s} t) / \varepsilon^{2}\right)}^{h^{+}(\mathbf{x}) / \varepsilon}\left|f\left(\mathbf{x}, \frac{\mathbf{x}-\mathbf{s} t}{\varepsilon^{2}}, Y\right)\right|^{2} \mathrm{~d} Y\right) \mathrm{d} \mathbf{x} \\
& \leq \varepsilon^{2} \int_{\mathbb{T}^{d}}\left(\int_{\mathbb{R}^{\prime}}\left|f\left(\mathbf{x}, \frac{\mathbf{x}-\mathbf{s} t}{\varepsilon^{2}}, Y\right)\right|^{2} \mathbf{1}_{\left[Y>-h^{-}\left((\mathbf{x}-\mathbf{s} t) / \varepsilon^{2}\right)\right]}(Y) \mathrm{d} Y\right) \mathrm{d} \mathbf{x}
\end{aligned}
$$

Now considering the function $\mathcal{F}(\mathbf{x}, \mathbf{X})=\int_{\mathbb{R}}|f(\mathbf{x}, \mathbf{X}, Y)|^{2} \mathbf{1}_{\left[Y>-h^{-}(\mathbf{X})\right]}(Y) \mathrm{d} Y$, we use a straightforward adaptation of Theorem 2 in [20] to obtain:

$$
\int_{\mathbb{T}^{d}}|\mathcal{F}(\mathbf{x}, \mathbf{x} / \varepsilon)| \mathrm{d} \mathbf{x} \leq \int_{\mathbb{T}^{d}} \sup _{\mathbf{X} \in \mathbb{T}^{d}}|\mathcal{F}(\mathbf{x}, \mathbf{X})| \mathrm{d} \mathbf{x} .
$$

By periodicity with respect to the second variable, the same argument applies to function $(\mathbf{x}, \mathbf{X}) \mapsto \mathcal{F}\left(\mathbf{x}, \mathbf{X}-\mathbf{s} t / \varepsilon^{2}\right)$ so that, defining the constant

$$
C(f):=\int_{\mathbb{T}^{d}}\left(\left.\sup _{\mathbf{X} \in \mathbb{T}^{d}}\left|\int_{\mathbb{R}}\right| f(\mathbf{x}, \mathbf{X}, Y)\right|^{2} \mathbf{1}_{\left[Y>-h^{-}(\mathbf{X})\right]}(Y) \mathrm{d} Y \mid\right) \mathrm{d} \mathbf{x},
$$

we obtain the following $L^{2}$-bound: $\left\|f^{\varepsilon}\right\|_{L^{2}\left(\Omega_{\varepsilon}(t)\right)}^{2} \leq \varepsilon^{2} C(f)$.

In order to state the $H^{1}$-estimates, we proceed as follows: first we have

$$
\begin{gathered}
\nabla_{\mathbf{x}} f^{\varepsilon}(\mathbf{x}, y, t)=\nabla_{\mathbf{x}} f\left(\mathbf{x}, \frac{\mathbf{x}-\mathbf{s} t}{\varepsilon^{2}}, \frac{y}{\varepsilon^{2}}\right)+\frac{1}{\varepsilon^{2}} \nabla_{\mathbf{x}} f\left(\mathbf{x}, \frac{\mathbf{x}-\mathbf{s} t}{\varepsilon^{2}}, \frac{y}{\varepsilon^{2}}\right), \\
\partial_{y} f^{\varepsilon}(\mathbf{x}, y, t)=\frac{1}{\varepsilon^{2}} \partial_{Y} f\left(\mathbf{x}, \frac{\mathbf{x}-\mathbf{s} t}{\varepsilon^{2}}, \frac{y}{\varepsilon^{2}}\right) .
\end{gathered}
$$

Then we apply the previous computation related to the $L^{2}$-estimate in order to get:

$$
\left\|\nabla_{\mathbf{x}, y} f^{\varepsilon}\right\|_{L^{2}\left(\Omega_{\varepsilon}(t)\right)}^{2}=\left\|\nabla_{\mathbf{x}} f^{\varepsilon}\right\|_{L^{2}\left(\Omega_{\varepsilon}(t)\right)}^{2}+\left\|\partial_{y} f^{\varepsilon}\right\|_{L^{2}\left(\Omega_{\varepsilon}(t)\right)}^{2} .
$$

Using the formula $(a+b)^{2} \leq 2\left(a^{2}+b^{2}\right)$ for the derivative with respect to $\mathbf{x}$, and using the previous $L^{2}$-estimate, we deduce:

$$
\left\|\nabla_{\mathbf{x}, y} f^{\varepsilon}\right\|_{L^{2}\left(\Omega_{\varepsilon}(t)\right)}^{2} \leq 2 \varepsilon^{2} C\left(\nabla_{\mathbf{x}} f\right)+2 \varepsilon^{-2} C\left(\nabla_{\mathbf{X}} f\right)+\varepsilon^{-2} C\left(\partial_{Y} f\right) .
$$




\section{E Proof of Proposition 4.5}

By means of a simple calculation, we get

$$
\begin{aligned}
\left\|g^{\varepsilon}\right\|_{L^{2}\left(\Omega_{\varepsilon}(t)\right)}^{2} & =\int_{\mathbb{T}^{d}}\left(\int_{-\varepsilon^{2} h^{-}\left((\mathbf{x}-\mathbf{s} t) / \varepsilon^{2}\right)}^{\varepsilon h^{+}(\mathbf{x})}\left|g\left(\mathbf{x}, \frac{y}{\varepsilon}\right)\right|^{2} \mathrm{~d} y\right) \mathrm{d} \mathbf{x} \\
& =\varepsilon \int_{\mathbb{T}^{d}}\left(\int_{-\varepsilon h^{-}\left((\mathbf{x}-\mathbf{s} t) / \varepsilon^{2}\right)}^{h^{+}(\mathbf{x})}|g(\mathbf{x}, Z)|^{2} \mathrm{~d} Z\right) \mathrm{d} \mathbf{x} \\
& =\varepsilon \int_{\mathbb{T}^{d}}\left(\int_{0}^{h^{+}(\mathbf{x})}|g(\mathbf{x}, Z)|^{2} \mathrm{~d} Z\right) \mathrm{d} \mathbf{x}+\mathcal{O}\left(\varepsilon^{2}\right),
\end{aligned}
$$

which states the result.

\section{F $\quad$ Proof of Lemma 4.8}

First, we infer from [12] the following property of the unfolding operator:

Proposition F.1 One has:

i) For any $f, g \in L^{2}\left(\mathbb{T}^{d} \times\right] a, b[)$ we have $\mathfrak{U}_{\delta}(f g)=\mathfrak{U}_{\delta}(f) \mathfrak{U}_{\delta}(g)$.

ii) For any $f \in L^{1}\left(\mathbb{T}^{d} \times\right] a, b[)$,

$$
\int_{\mathbb{T}^{d}} \int_{a}^{b} f(\mathbf{x}, y) \mathrm{d} y \mathrm{~d} \mathbf{x}=\int_{\mathbb{T}^{d}} \int_{\mathbb{T}^{d}} \int_{a}^{b} \mathfrak{U}_{\delta}(f)(\mathbf{x}, \mathbf{X}, y) \mathrm{d} y \mathrm{~d} \mathbf{X} \mathrm{d} \mathbf{x} .
$$

Using this proposition, we prove Lemma $4.8 i$ ) (other items may be proven with a straightforward computation). We have: $\|f\|_{L^{2}\left(\Omega_{\varepsilon}\right)}^{2}=\|f\|_{L^{2}\left(\Omega_{\varepsilon}^{+}\right)}^{2}+\|f\|_{L^{2}\left(\Omega_{\varepsilon}^{-}\right)}^{2}$. Using the properties of the rescaling operator,

$$
\begin{aligned}
\|f\|_{L^{2}\left(\Omega_{\varepsilon}^{+}\right)}^{2} & :=\int_{\mathbb{T}^{d}} \int_{0}^{\varepsilon h^{+}(\mathbf{x})}|f(\mathbf{x}, y)|^{2} \mathrm{~d} y \mathrm{~d} \mathbf{x}=\varepsilon \int_{\mathbb{T}^{d}} \int_{0}^{h^{+}(\mathbf{x})}|f(\mathbf{x}, \varepsilon Z)|^{2} \mathrm{~d} Z \mathrm{~d} \mathbf{x} \\
& =\varepsilon \int_{\mathbb{T}^{d}} \int_{0}^{h^{+}(\mathbf{x})}\left|\Re_{\varepsilon}(f)(\mathbf{x}, Z)\right|^{2} \mathrm{~d} Z \mathrm{~d} \mathbf{x}
\end{aligned}
$$

where we have used (a) the change of variables $y=\varepsilon Z$, (b) the definition of the rescaling operator. Using the properties of the rescaling and unfolding 
operators,

$$
\begin{aligned}
\|f\|_{L^{2}\left(\Omega_{\varepsilon}^{-}\right)}^{2} & :=\int_{\mathbb{T}^{d}} \int_{-\varepsilon^{2} h^{-}\left(\frac{\mathbf{x}}{\varepsilon^{2}}\right)}^{0}|f(\mathbf{x}, y)|^{2} \mathrm{~d} y \mathrm{~d} \mathbf{x} \\
& =\int_{\mathbb{T}^{d}} \int_{\mathbb{R}^{\mathbb{R}}}|f(\mathbf{x}, y)|^{2} \mathbf{1}_{]-\varepsilon^{2} h^{-}\left(\frac{\mathbf{x}}{\varepsilon^{2}}\right), 0[}(y) \mathrm{d} y \mathrm{~d} \mathbf{x} \\
& =\int_{\mathbb{T}^{d}} \int_{\mathbb{T}^{d}} \mathcal{U}_{\varepsilon^{2}}\left(\int_{\mathbb{R}}|f(\cdot, y)|^{2} \mathbf{1}_{]-\varepsilon^{2} h^{-}\left(\frac{\mathbf{x}}{\varepsilon^{2}}\right), 0[}(y) \mathrm{d} y\right)(\mathbf{x}, \mathbf{X}) \mathrm{d} \mathbf{X} \mathrm{d} \mathbf{x} \\
& =\int_{\mathbb{T}^{d}} \int_{\mathbb{T}^{d}}\left(\int_{\mathbb{R}}\left|\mathfrak{U}_{\varepsilon^{2}}(f)\right|^{2}(\mathbf{x}, \mathbf{X}, y) \mathbf{1}_{]-\varepsilon^{2} h^{-}(\mathbf{X}), 0[}(y) \mathrm{d} y\right) \mathrm{d} \mathbf{X} \mathrm{d} \mathbf{x} \\
& =\int_{\mathbb{T}^{d}} \int_{\mathbb{T}^{d}}\left(\int_{-\varepsilon^{2} h^{-}(\mathbf{X})}^{0}\left|\mathfrak{U}_{\varepsilon^{2}}(f)\right|^{2}(\mathbf{x}, \mathbf{X}, y) \mathrm{d} y\right) \mathrm{d} \mathbf{X} \mathrm{d} \mathbf{x} \\
& =\varepsilon^{2} \int_{\mathbb{T}^{d}} \int_{\mathbb{T}^{d}}\left(\int_{-h^{-}(\mathbf{X})}^{0}\left|\mathfrak{U}_{\varepsilon^{2}}(f)\right|^{2}\left(\mathbf{x}, \mathbf{X}, \varepsilon^{2} Y\right) \mathrm{d} Y\right) \mathrm{d} \mathbf{X} \mathrm{d} \mathbf{x} \\
& =\varepsilon^{2} \int_{\mathbb{T}^{d}} \int_{\mathbb{T}^{d}}\left(\int_{-h^{-}(\mathbf{X})}^{0}\left|\mathfrak{R}_{\varepsilon^{2}} \circ \mathfrak{U}_{\varepsilon^{2}}(f)\right|^{2}(\mathbf{x}, \mathbf{X}, Y) \mathrm{d} Y\right) \mathrm{d} \mathbf{X} \mathrm{d} \mathbf{x}
\end{aligned}
$$

where we have used (a) Proposition F.1-ii), (b) Proposition F.1-i), (c) the change of variables $y=\varepsilon^{2} Y$, (d) the definition of the rescaling operator.

\section{References}

[1] Youcef Amirat, Didier Bresch, Jérôme Lemoine, and Jacques Simon. Effect of rugosity on a flow governed by stationary Navier-Stokes equations. Quart. Appl. Math., 59(4):769-785, 2001.

[2] Youcef Amirat and Jacques Simon. Influence de la rugosité en hydrodynamique laminaire. C. R. Acad. Sci. Paris Sér. I Math., 323(3):313-318, 1996.

[3] Arnaud Basson and David Gérard-Varet. Wall laws for fluid flows at a boundary with random roughness. Comm. Pure Appl. Math., 61(7):941987, 2008.

[4] Guy Bayada and Michèle Chambat. The transition between the Stokes equations and the Reynolds equation: a mathematical proof. Appl. Math. Optim., 14(1):73-93, 1986.

[5] Nadia Benhaboucha, Michèle Chambat, and Ionel Ciuperca. Asymptotic behaviour of pressure and stresses in a thin film flow with a rough boundary. Quart. Appl. Math., 63(2):369-400, 2005.

[6] Mikhail E. Bogovski1. Solution of the first boundary value problem for an equation of continuity of an incompressible medium. Dokl. Akad. Nauk SSSR, 248(5):1037-1040, 1979. 
[7] Mahdi Boukrouche and Ionel Ciuperca. Asymptotic behaviour of solutions of lubrication problem in a thin domain with a rough boundary and Tresca fluid-solid interface law. Quart. Appl. Math., 64(3):561-591, 2006.

[8] Franck Boyer and Pierre Fabrie. Éléments d'analyse pour l'étude de quelques modèles d'écoulements de fluides visqueux incompressibles, volume 52 of Mathématiques \&S Applications (Berlin) [Mathematics $\&$ Applications]. Springer-Verlag, Berlin, 2006.

[9] Didier Bresch, Catherine Choquet, Laurent Chupin, Thierry Colin, and Marguerite Gisclon. Roughness-induced effect at main order on the Reynolds approximation. Multiscale Model. Simul., 8(3):997-1017, 2010.

[10] Didier Bresch and Vuk Milisic. High order multi-scale wall-laws, Part I: the periodic case. Quart. Appl. Math., 68(2):229-253, 2010.

[11] Juan Casado-Díaz, Manuel Luna-Laynez, and Francisco Javier SuárezGrau. Asymptotic behavior of a viscous fluid with slip boundary conditions on a slightly rough wall. Math. Models Methods Appl. Sci., 20(1):121-156, 2010 .

[12] Doina Cioranescu, Alain Damlamian, and Georges Griso. The periodic unfolding method in homogenization. SIAM J. Math. Anal., 40(4):15851620, 2008.

[13] Harold G. Elrod. Thin-film lubrication theory for Newtonian fluids with surfaces possessing striated roughness or grooving. ASME J. Lubr. Technol., 95:484-489, 1973.

[14] Giovanni P. Galdi. An introduction to the mathematical theory of the Navier-Stokes equations. Vol. I, volume 38 of Springer Tracts in Natural Philosophy. Springer-Verlag, New York, 1994. Linearized steady problems.

[15] David Gérard-Varet. The Navier wall law at a boundary with random roughness. Comm. Math. Phys., 286(1):81-110, 2009.

[16] Willi Jäger and Andro Mikelić. On the boundary conditions at the contact interface between a porous medium and a free fluid. Ann. Scuola Norm. Sup. Pisa Cl. Sci. (4), 23(3):403-465, 1996.

[17] Willi Jäger and Andro Mikelić. On the interface boundary condition of Beavers, Joseph, and Saffman. SIAM J. Appl. Math., 60(4):1111-1127 (electronic), 2000.

[18] Willi Jäger and Andro Mikelić. On the roughness-induced effective boundary conditions for an incompressible viscous flow. J. Differential Equations, 170(1):96-122, 2001.

[19] Jean-Louis Ligier. Lubrification des paliers moteurs. Technip, 1997. 
[20] Dag Lukkassen, Gabriel Nguetseng, and Peter Wall. Two-scale convergence. Int. J. Pure Appl. Math., 2(1):35-86, 2002.

[21] Claude Louis Marie Henri Navier. Sur les lois de l'équilibre et du mouvement des corps élastiques. Mem. Acad. R. Sci. Inst. France, 6(369), 1827.

[22] Sergueï A. Nazarov and Juha H. Videman. A modified nonlinear Reynolds equation for thin viscous flows in lubrication. Asymptot. Anal., 52(1-2):1$36,2007$.

[23] Nhan Phan-Thien. On the effects of the Reynolds and Stokes surface roughnesses in a two-dimensional slider bearing. Proc. R. Soc. Lond. A, 377:349$362,1981$.

[24] Osborne Reynolds. On the theory of lubrication and its application to $\mathrm{Mr}$ Beauchamp tower's experiments, including an experimental determination of the viscosity of olive oil. Phil. Trans. Roy. Soc. London, A117:157-234, 1886.

[25] Enrique Sánchez-Palencia. Nonhomogeneous media and vibration theory, volume 127 of Lecture Notes in Physics. Springer-Verlag, Berlin, 1980.

[26] Dah-chen Sun and Kuo-Kuang Chen. First effects of Stokes roughness on hydrodynamic lubrication. ASME J. Lubr. Technol., 99(1):2-9, 1977.

[27] Jon Wilkening. Practical error estimates for Reynolds' lubrication approximation and its higher order corrections. SIAM J. Math. Anal., 41(2):588$630,2009$. 